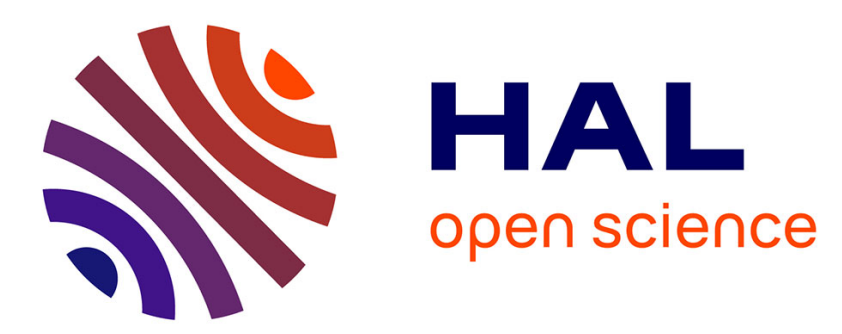

\title{
A model for the dynamics of crater-centered intrusion: Application to lunar floor-fractured craters
}

Clément Thorey, Chloé Michaut

\section{To cite this version:}

Clément Thorey, Chloé Michaut. A model for the dynamics of crater-centered intrusion: Application to lunar floor-fractured craters. Journal of Geophysical Research. Planets, 2014, 119, pp.286-312. 10.1002/2013JE004467 . insu-03581156

\section{HAL Id: insu-03581156 https://hal-insu.archives-ouvertes.fr/insu-03581156}

Submitted on 19 Feb 2022

HAL is a multi-disciplinary open access archive for the deposit and dissemination of scientific research documents, whether they are published or not. The documents may come from teaching and research institutions in France or abroad, or from public or private research centers.
L'archive ouverte pluridisciplinaire HAL, est destinée au dépôt et à la diffusion de documents scientifiques de niveau recherche, publiés ou non, émanant des établissements d'enseignement et de recherche français ou étrangers, des laboratoires publics ou privés.

$$
\text { Copyright }
$$


Journal of Geophysical Research: Planets

\section{RESEARCH ARTICLE}

10.1002/2013JE004467

Key Points:

- A model for the dynamics of crater-centered intrusion is proposed

- Diverse floor appearances are obtained and correspond to observations

- The floor appearance depends on the intrusion overlying layer properties

Correspondence to:

C. Thorey,

thorey@ipgp.fr

Citation:

Thorey, C., and C. Michaut (2014),

A model for the dynamics of crater-centered intrusion: Application to lunar floor-fractured craters, $J$. Geophys. Res. Planets, 119, 286-312, doi:10.1002/2013JE004467.

Received 19 JUN 2013

Accepted 28 DEC 2013

Accepted article online 4 JAN 2014

Published online 31 JAN 2014

\section{A model for the dynamics of crater-centered intrusion: Application to lunar floor-fractured craters}

\author{
Clément Thorey ${ }^{1}$ and Chloé Michaut ${ }^{1}$ \\ 'Sciences Spatiales et Planétologie, Université Paris Diderot, Sorbonne Paris Cité, Institut de Physique du globe de Paris, \\ UMR 7154 CNRS, F-75013 Paris, France
}

\begin{abstract}
Lunar floor-fractured craters are a class of craters modified by post-impact mechanisms. They are defined by distinctive shallow floors that are convex or plate-like, sometimes with a wide floor moat bordering the wall region. Radial, concentric, and polygonal floor fractures suggest an endogenous process of modification. Two mechanisms have been proposed to account for such deformations: viscous relaxation and spreading of a magma intrusion at depth below the crater. To test the second assumption and bring more constraints on the intrusion process, we develop a model for the dynamics of magma spreading below an elastic overlying layer with a crater-like topography. As predicted in earlier more qualitative studies, the increase in lithostatic pressure at the crater wall zone prevents the intrusion from spreading laterally, leading to the thickening of the intrusion. Additionally, our model shows that the final crater floor appearance after the uplift, which can be convex or flat, with or without a circular moat bordering the wall zone, depends on the elastic thickness of the layer overlying the intrusion and on the crater size. Our model provides a simple formula to derive the elastic thickness of the overlying layer hence a minimum estimate for the intrusion depth. Finally, our model suggests that crust redistribution by cratering must have controlled magma ascent below most of these craters.
\end{abstract}

\section{Introduction}

A large fraction of the magma produced by mantle melting never reaches the surface as it intrudes the shallow layers of the planet. On Earth, the volume of intrusive magma is estimated to be 10 times (respectively, 5 times) the volume of extrusive lava for the continental crust (respectively, the oceanic crust) [Crisp, 1984]. Buoyancy is the main mechanism driving up the magma from the interior to the shallow layers or the surface of the planets. It has been shown that dykes stop their propagation when they become neutrally buoyant relative to their surroundings [Walker, 1989; Rivalta et al., 2005; Taisne and Tait, 2009]. Therefore, the intrusive to extrusive ratio largely depends on the respective density of the crust and magma.

The density of the lunar crust is particularly low. The last estimate from the Gravity Recovery and Interior Laboratory NASA mission provides for a mean density for the highlands of $2550 \mathrm{~kg} \mathrm{~m}^{-3}$, even lower than what was previously assumed [Wieczorek et al., 2013]. Both the light anorthite minerals that form the lunar crust and impact induced fractures and brecciation contribute to its low density [Wilhelms et al., 1987]. Given the large density of magma inferred from the composition of the mare basalts that are generally rich in FeO and $\mathrm{TiO}_{2}$ [Wieczorek et al., 2001], the intrusive to extrusive ratio on the Moon might be even higher than on Earth [El-Baz, 1970; Wilson and Head, 1981; Hiesinger and Head, 2006; Glotch et al., 2010]. Head and Wilson [1992] estimated an upper limit of 50:1 for this ratio. However, there are no solid constraints supporting this estimate although it is an important parameter in lunar thermal evolution models [Laneuville et al., 2013].

Extrusions of lava preferentially occurred within large impact basins on the near side of the Moon where a large fraction of the low-density crust has been removed. However, the trajectory of the magma from its source to the surface is unknown. The magma could have ascended directly from the source to the surface [Wieczorek et al., 2001]; alternatively, it might have first accumulated at the crust-mantle interface before erupting where the crust was thinner [Wilson and Head, 1981].

Possible sites for intrusions on the Moon are below floor-fractured craters (FFCs). These craters have been identified by Schultz [1976a]; they have shallow floors with a plate-like or convex appearance, wide floor moats, and radial, concentric, and polygonal floor fractures. Schultz [1976a] has classified about 200 FFCs. Similar FFCs have been observed on Mercury [Head et al., 2009], Mars [Schultz, 1978; Schultz and Glicken, 
1979; Sato et al., 2010], and Venus [Wichman and Schultz, 1995a]. The database and classification proposed by Schultz [1976a] have recently been updated by Jozwiak et al. [2012] who used new data from the Lunar Orbiter Laser Altimeter (LOLA) and Lunar Reconnaissance Orbiter Camera (LROC). The deformations affecting these craters are contained within the crater interior. Two mechanisms have been proposed to explain the features observed at FFCs: (1) spreading of a magmatic intrusion at depth below the crater floor [Schultz, 1976a; Wichman and Schultz, 1993, 1995b, 1996; Jozwiak et al., 2012] and (2) viscous relaxation of the crater floor induced by a local thermal gradient caused by the impact [Hall et al., 1981]. However, viscous relaxation of the crater floor has been modeled by Dombard and Gillis [2001], but for typical elastic parameters, the lunar crust is too rigid and shallowing of craters smaller than $100 \mathrm{~km}$ is not significant. Hence, if the deformations observed at FFCs resulted indeed from magmatic intrusion, they might give us important constraints and clues on the process of magmatic intrusion in the lunar crust.

The classical, static, model of laccoliths of Pollard and Johnson [1973] has previously been applied to the case of FFCs to deduce the intrusion depth and magma pressure [Wichman and Schultz, 1993, 1996; Jozwiak et al., 2012]. In this static model, the intrusion radius is known a priori. The intrusion shape is controlled by the elastic deformation of a thin elastic layer on top of the laccolith, and the elastic pressure necessary for deforming the overlying layer is assumed to be equilibrated by magma weight. However, Michaut [2011] and Bunger and Cruden [2011] have shown that if the elastic deformation of the overlying layer controls the flow shape and its dynamics during a first spreading phase, the weight of the flow itself becomes dominant during a second phase where the flow shape shows a flat top. Furthermore, in all these models, the possible effect of a thickening of the overlying layer has been ignored.

Here we modify the model proposed by Michaut [2011] for the dynamics of a magma intrusion below an elastic overlying layer in order to account for the effects of the crater topography, i.e., for an overlying layer of variable thickness. We show that different types of deformations of the crater floor are expected, as initially predicted by Schultz [1976a], and that they mainly depend on the elastic thickness of the layer overlying the intrusion and on the crater size.

\section{Floor-Fractured Craters}

Floor-fractured craters are craters that have undergone endogenous deformations after the impact. About 200 FFCs have been observed on the Moon and precisely described by Schultz [1976a] who studied their structure and geology using Lunar Orbiter and Apollo stereo sets. Schultz [1976a] proposed a classification into six categories based on their sizes, morphological features, and degrees of modification; classification which has recently been updated by Jozwiak et al. [2012] using the Lunar Orbiter Laser Altimeter (LOLA) and Lunar Reconnaissance Orbiter Camera (LROC) (Table 1).

Radial and concentric fracture networks generally cross the floors of these craters [Schultz, 1976a]. Another striking feature of FFCs is their shallow floors: except for class 1 FFCs, they all exhibit a significant shallowing of their floors compared to fresh craters the same size [Schultz, 1976a; Jozwiak et al., 2012].

Floor uplift mainly results in two different modes of crater floor appearance [Schultz, 1976a]. In particular, FFCs of classes 3 and 5 show a flat central floor, characteristic of a piston-like uplift of the crater floor; additionally, a large circular U-shaped moat adjacent to the wall zone borders the flat floor of class $3 \mathrm{FFCs}$ [Schultz, 1976a; Jozwiak et al., 2012]. A typical example of a class 3 FFC is the crater Warner, which is $35 \mathrm{~km}$ in diameter and is located at $4.0^{\circ} \mathrm{S}, 87.3^{\circ} \mathrm{E}$ in the southern part of the Mare Smythii (Figure $1 \mathrm{~b}$ ). In contrast, the floors of craters of classes 2 and 4 appear convex, indicating a different mechanism of crater floor uplift [Schultz, 1976a; Jozwiak et al., 2012]. Briggs is a good archetype of class 2 FFC, it is a crater of $37 \mathrm{~km}$ in diameter and is located at $26.5^{\circ} \mathrm{N}, 69.1^{\circ} \mathrm{W}$ in the western part of the Oceanus Procellarum (Figure 1a). Craters showing a convex floor may also exhibit moats adjacent to their wall zone, but these are V-shaped; this is the case for classes $4 a, 4 b$, and 4c FFCs. In addition, craters of class $4 \mathrm{~b}$ also exhibit an inner wall zone.

Finally, class 1 FFCs show only limited deformations while the floor of class 6 FFCs has been flooded by mare lavas, illustrating the close relationship between magmatism and deformations at these craters [Schultz, 1976a; Jozwiak et al., 2012]. 


\begin{tabular}{|c|c|}
\hline Class & Description \\
\hline 1 & $\begin{array}{c}\text { Large craters: } 50-300 \mathrm{~km} \text { (average } 140 \mathrm{~km} \text { ) } \\
\text { Deep floor } \\
\text { Absence of moats } \\
\text { Radial or concentric fractures }\end{array}$ \\
\hline 2 & $\begin{array}{c}\text { Midsize craters: } 13-75 \mathrm{~km} \text { (average } 30 \mathrm{~km} \text { ) } \\
\text { Shallow convex floor } \\
\text { Absence of moats } \\
\text { Strong concentric fractures }\end{array}$ \\
\hline 3 & $\begin{array}{l}\text { 12-170 km (average } 50 \mathrm{~km} \text { ) } \\
\text { Shallow central flat floor } \\
\text { Wide U-shaped moat } \\
\text { Radial or polygonal fractures }\end{array}$ \\
\hline $4 a$ & $\begin{array}{l}\text { Small craters: 4-38 km (average } 15 \mathrm{~km} \text { ) } \\
\text { Shallow convex floor } \\
\text { Weak V-shaped moat } \\
\text { Strong radial and concentric fractures }\end{array}$ \\
\hline $4 b$ & $\begin{array}{l}\text { Small craters: } 7-45 \mathrm{~km} \text { (average } 25 \mathrm{~km} \text { ) } \\
\text { Shallow convex floor } \\
\text { Pronounced V-shaped moat }+ \text { inner ridges } \\
\text { Subtle radial fractures }\end{array}$ \\
\hline $4 c$ & $\begin{array}{l}\text { Small craters: average } 15 \mathrm{~km} \\
\text { Flat or concave up floor } \\
\text { V-shaped moats } \\
\text { Hummocky, lack of fracture }\end{array}$ \\
\hline 5 & $\begin{array}{l}\text { Large craters: } 12-177 \mathrm{~km} \text { (average } 70 \mathrm{~km} \text { ) } \\
\text { Shallow old and degraded central flat floor } \\
\text { Absence of moats } \\
\text { Strong radial, concentric and/or polygonal fractures }\end{array}$ \\
\hline 6 & $\begin{array}{l}\text { Large craters: } 50-200 \mathrm{~km} \\
\text { Completely flooded by basalt } \\
\text { Absence of moats } \\
\text { Concentric fractures }\end{array}$ \\
\hline
\end{tabular}

\section{An Axisymmetric Model for a Mag- matic Intrusion Spreading Below a Crater-Like Topography}

In this model, we consider the spreading of an axisymmetric intrusion above a rigid layer and below a thin overlying layer with a crater-like topography.

\subsection{Crater Topography and Overlying Layer Characteristics}

On the Moon, fresh impact craters have been classified into three categories according to their shapes following crater formation and collapse: simple craters, complex craters, and basins [Pike, 1974; Schultz, 1976b; Pike, 1980; Baker et al., 2012]. Simple craters are bowl-shaped craters that do not exhibit any slope-break [Pike, 1980]. With increasing diameter, impact craters transition to complex craters that are characterized by an inner flat floor, terraced rims, and a central peak. On the Moon, the transition from simple to complex craters occurs at a diameter of $15 \mathrm{~km}$ [Pike, 1980; Hiesinger and Head, 2006; O'Keefe and Ahrens, 1999; Kalynn et al., 2013]. Although not all do, most craters larger than $100 \mathrm{~km}$ exhibit rings on their flat floors and are defined as basins [Wilhelms et al., 1987; Schultz, 1988]. In our model, we do not consider basins and study the spreading of a magmatic intrusion at depth below simple and complex craters.

In this model, we account for the effects of the crater topography on the intrusion dynamics. The normalized sigmoid function $\xi(r)$ is used to parameterize the crater topography:

$$
\xi(r)=\frac{1}{1+\mathrm{e}^{-\frac{4 \alpha(r-C)}{D_{C}}}}-\frac{1}{1+\mathrm{e}^{\frac{4 \alpha C}{D_{C}}}}
$$

where $D_{c}$ is the intrusion depth, $C$ the crater radius, defined as the distance from the crater center to the center of the wall zone, and $\alpha$ the average slope of the wall zone (Figure 2). The elevation in height relative to the crater center, i.e., the crater topography, is then given by

$$
T_{p}(r)=D_{c} \xi(r)
$$

and results in an increase in lithostatic pressure at the crater wall zone.

For simple craters, our definition of the crater radius $C$ corresponds to half of the observed crater radius. Pike [1980] shows that the average wall slope $\alpha$ increases gradually from about $0.3\left(19^{\circ}\right)$, for craters $0.5 \mathrm{~km}$ in diameter, to $0.4\left(25^{\circ}\right)$ for craters $15 \mathrm{~km}$ in diameter [Kalynn et al., 2013]. To model a simple crater, we thus use $D_{c} / 4 \alpha C=0.25$ (Figure 2a), which, for $C=2.5 \mathrm{~km}$ and $\alpha=0.4\left(25^{\circ}\right)$ gives a corresponding $D_{c}$ of $1 \mathrm{~km}$ in agreement with Pike [1980]. For complex craters, the size of the central flat floor, relatively to the crater radius, increases from $25 \%$, for a crater $20 \mathrm{~km}$ in diameter, to $50 \%$, for a crater that is $100 \mathrm{~km}$ in diameter. The wall of complex craters exhibits walls at the angle of repose $\left(\sim 30^{\circ}\right)$, terraces, and hilly toes that combine to form a wall zone of effective slope value that decreases from $\alpha=0.5\left(30^{\circ}\right)$ to $\alpha=0.2\left(12^{\circ}\right)$ as the crater diameter increases from 20 to 100 km [Pike, 1980; Bray et al., 2008; Kalynn et al., 2013]. To model a complex crater, we use a ratio $D_{c} / 4 \alpha C=0.13$ (Figure $2 \mathrm{~b}$ ) which, for $C=14 \mathrm{~km}$ and $\alpha=0.4$, i.e., a crater that is $40 \mathrm{~km}$ in diameter, gives $D_{c}=3 \mathrm{~km}$ in agreement with Pike [1980] and Kalynn et al. [2013]. 
a) Crater Briggs, $26.5^{\circ} \mathrm{N} 69.1^{\circ} \mathrm{W}$

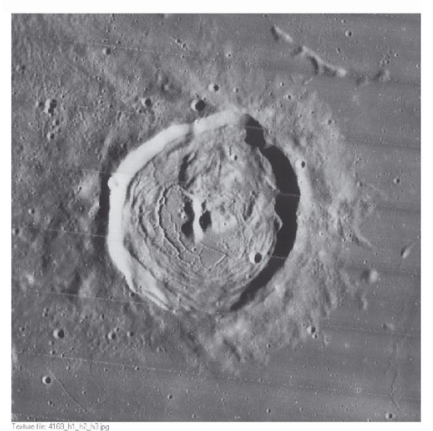

b) Crater Warner, $4,0^{\circ} \mathrm{S} 87.3^{\circ} \mathrm{W}$

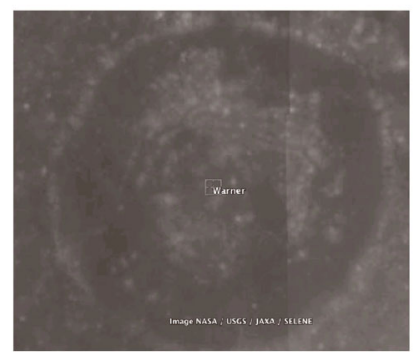

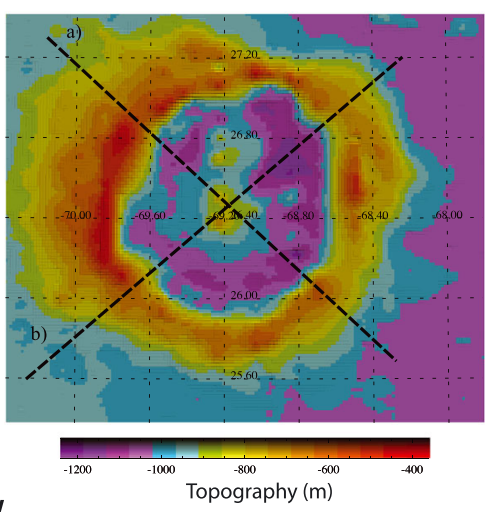

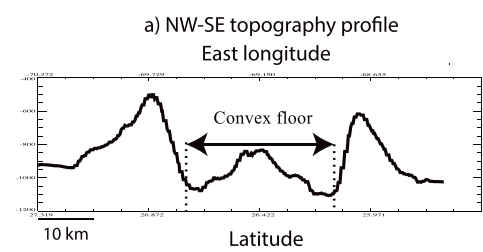

b) SW-NE topography profile East longitude

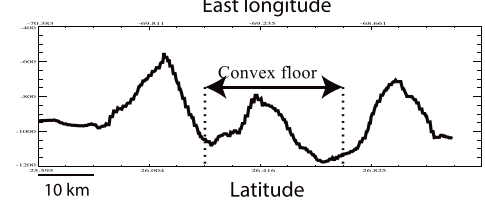

a) N-S topography profile East longitude
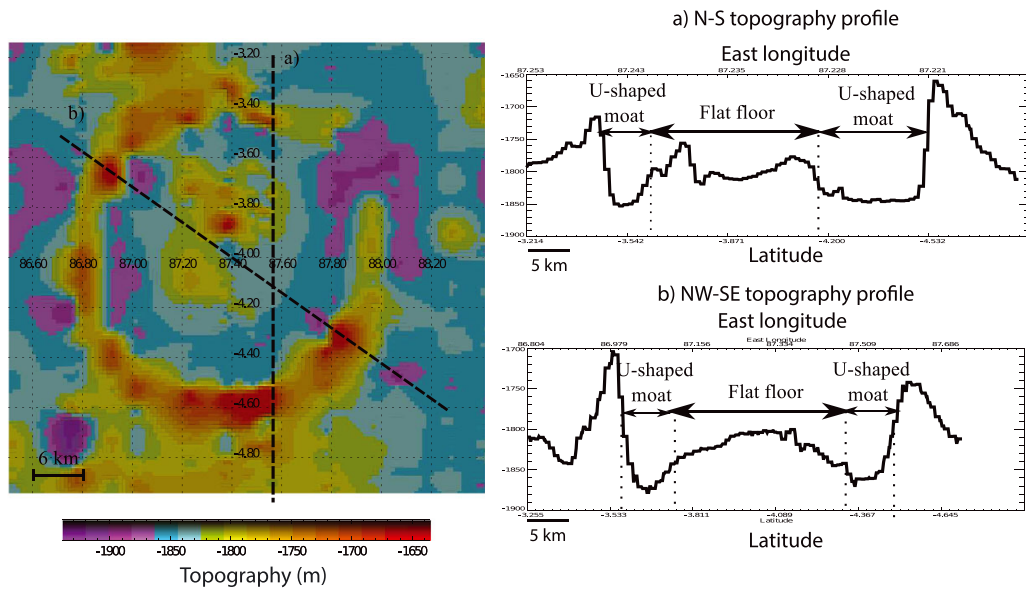

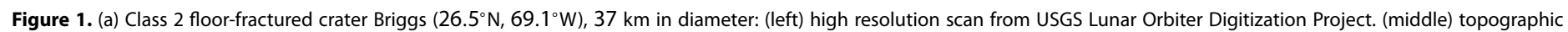

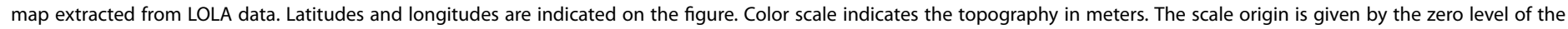

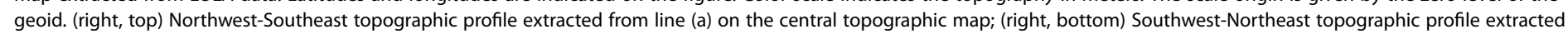
from line (b) on the central topographic map. (b) Same plots for the class $3 \mathrm{FFC}$ Warner $\left(4.0^{\circ} \mathrm{S}, 87.3^{\circ} \mathrm{E}\right)$ that is $35 \mathrm{~km}$ in diameter.

Impact induces fracture and brecciation beneath the crater floor [Wilhelms et al., 1987; Melosh, 1989; Jolliff et al., 2000] and also causes melting and compaction of the pores [Melosh, 1989; Schultz, 1976a]. If the melting during impact is negligible and the neutral buoyancy zone of the magma lies immediately beneath an impact brecciated lens, as is commonly assumed [Schultz, 1976a; Wichman and Schultz, 1996; Jozwiak et al., 2012], the overlying layer would not respond elastically due to its lack of coherent structure. We consider the case of a strengthless overlying layer with a crater-like topography given by (2) in section 4.1. However, a coherent impact melt unit commonly stands on top of the brecciated lens [Melosh, 1989; Schultz, 1976a]. The neutral buoyancy zone of the magma depends on the crust and magma density and could also be situated deeper than the bottom of the brecciated lens. As a result, the overlying layer would deform elastically above the intrusion. In our model, we thus consider the case of an overlying layer with an elastic thickness $T_{e}(r)$ that varies with radial coordinate $r$ and thickens at the crater wall zone (Figure 3). The elastic layer is characterized by a Young's modulus $E$ and a Poisson's ratio $v$. For simplicity, the thickness of the elastic layer, showing a simple or a complex crater topography, is considered to be equal to the intrusion depth and is given by

$$
T_{e}(r)=T_{e}^{0}(1+\Psi \xi(r))
$$

where $\Psi=D_{c} / T_{e}^{0}$ is the ratio of the crater depth $D_{c}$ to the intrusion depth at the center $T_{e}^{0}$ and characterizes the thickening of the upper elastic layer at the crater wall zone. As a result of this assumption, the intrusion 

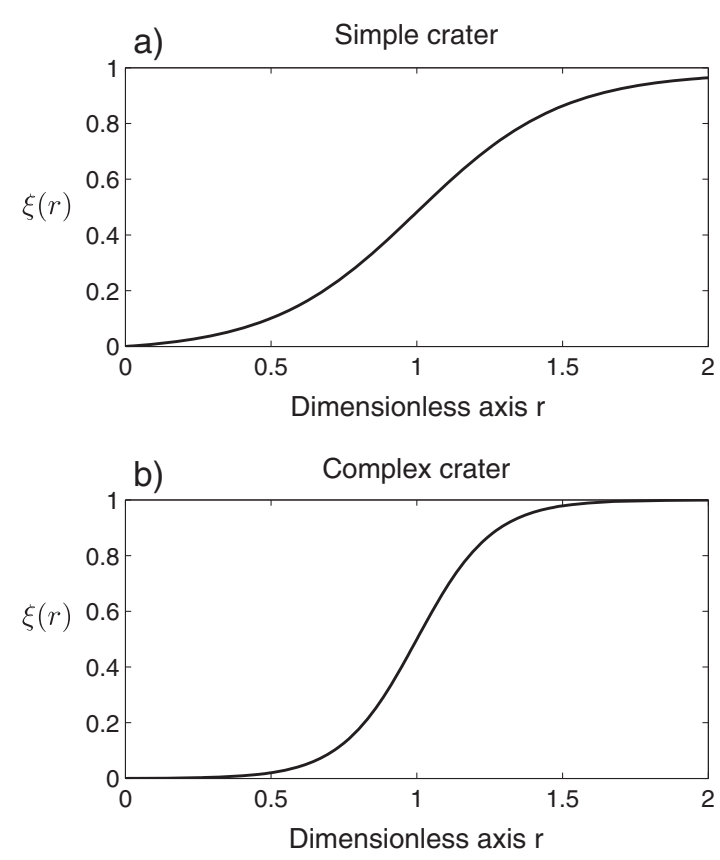

Figure 2. Here we show the two normalized sigmoid function $\xi(r)$ used to parameterize the two end-member crater topographies (see section 3.1). (a) The sigmoid function of a simple crater modeled using $D_{c} / 4 \alpha C=\zeta=0.25$. (b) The sigmoid function of a complex crater modeled using $D_{c} / 4 \alpha C=\zeta=0.13$. depth is underestimated in the model, but if a constant thickness-brecciated layer overlies the intrusion and underlies such an elastic layer, it does not have any effect on the intrusion dynamics. The crater topography $T_{p}$ is then given by

$$
T_{p}(r)=T_{e}(r)-T_{e}^{0}=T_{e}^{0} \Psi \xi(r)=D_{c} \xi(r)
$$

Central peaks are common features of complex craters. Their dimensions depend on both projectile and target properties as well as on the impact angle [Schultz et al., 1994; Bray et al., 2008]. However, their width can reach one fourth of the crater floor and they can be as high as half of the crater depth; hence, such a structure could influence the intrusion dynamics. The effect of a central peak on the spreading of an intrusion is examined in Appendix C. Moreover, a raised rim, uplifted relative to the preimpact surface, is usually present at the exterior of the crater wall zone [Pike, 1976, 1980]. Although, for simplicity, we do not model the effect of this feature, we discuss its possible influence on intrusion emplacement in section 5.3.

\subsection{Equations of Motion}

We assume that the flow spreads along a thin bedding plane and neglect fracturing at the tip. The magma is considered to behave as a Newtonian fluid with a constant viscosity $\mu$ and density $\rho_{m}$. The intrusion is fed at a constant rate through a cylindrical conduit of diameter $a$. The vertical coordinate $z$ is oriented upward (Figure 3).

\subsubsection{Momentum Equation}

The dynamics of the flow is given by the solution of the Navier-Stokes equation in cylindrical geometry. The magma has a relatively large viscosity, hence, the flow is in a laminar regime and the inertia terms can be neglected. The intrusion develops over a length scale comparable to the crater size that is much larger than its thickness $H(C>>H)$, hence, the lubrication assumption allows further simplifications [Huppert,

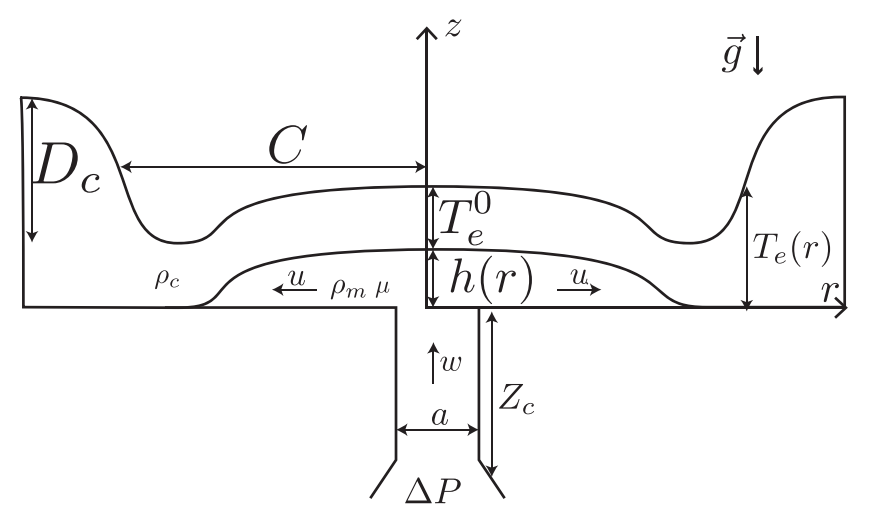

Figure 3. Sketch of the intrusion spreading below an impact crater topography. $D_{c}$ (respectively, $C$ ) represents the initial depth (respectively, radius) of the impact crater. The intrusion spreads above a rigid homogeneous and horizontal bedrock at depth $T_{e}(r)$ below the crater floor. At the center, the intrusion depth is noted $T_{e}^{0}$. 
1982; Michaut and Bercovici, 2009]. The Navier-Stokes equations in the radial and vertical directions for an axisymmetric, incompressible flow of Newtonian fluid reduce to

$$
\begin{array}{r}
-\frac{\partial P}{\partial r}+\mu \frac{\partial^{2} u}{\partial z^{2}}=0 \\
-\frac{\partial P}{\partial z}-\rho_{m} g=0
\end{array}
$$

Integration of (6) gives the pressure within the flow

$$
P(r, t)=\rho_{m} g(h(r, t)-z)+\rho_{c} g T_{e}(r)+P_{e}(r, t)
$$

where $h(r, t)$ is the intrusion thickness and $\rho_{c}$ is the overlying layer density. The pressure is the sum of three different contributions: the weight of the magma and of the overlying layer and the elastic pressure $P_{e}$ due to the deformation of the overlying elastic layer. In the absence of radial forces within the elastic plate, the elastic pressure required for bending the plate is given by the force per unit area that is necessary for a vertical displacement $h$ of the thin elastic plate [Turcotte et al., 1981]:

$$
P_{e}(r, t)=\nabla_{r}^{2}\left(D(r) \nabla_{r}^{2} h(r)\right)
$$

where

$$
D(r)=\frac{E T_{e}(r)^{3}}{12\left(1-v^{2}\right)}
$$

is the flexural rigidity of the plate which depends on the elastic layer thickness $T_{e}(r)$, Young's modulus $E$, and Poisson's ratio $v$.

Substituting (7) and (8) into (5) and integrating twice using no-slip boundary conditions at the top and bottom of the intrusion, i.e., $u_{z=0}=u_{z=h}=0$, we get the radial velocity within the flow:

$$
u(r, z, t)=\frac{1}{2 \mu}\left(\rho_{m} g \frac{\partial h(r, t)}{\partial r}+\rho_{c} g \frac{\partial T_{e}(r)}{\partial r}+\frac{\partial}{\partial r}\left(\nabla_{r}^{2}\left(D(r) \nabla_{r}^{2} h(r, t)\right)\right)\right)\left(z^{2}-z h(r, t)\right)
$$

\subsubsection{Injection Rate}

Assuming a Poiseuille flow within the cylindrical feeding conduit, the vertical injection velocity $w(r, t)$ and injection rate $Q_{0}$ are given by

$$
\begin{gathered}
w(r, t)= \begin{cases}\frac{\Delta P}{4 \mu z_{c}}\left(\frac{a^{2}}{4}-r^{2}\right) & r \leq \frac{a}{2} \\
0 & r>\frac{a}{2}\end{cases} \\
Q_{0}=\frac{\pi \Delta P a^{4}}{128 \mu Z_{c}}
\end{gathered}
$$

where $\Delta P$ is the initial overpressure within the melt at $z=Z_{c}$.

\subsubsection{Final Equation}

To obtain the final equation of motion, we write mass conservation integrated over the flow thickness:

$$
\frac{\partial h(r, t)}{\partial t}=-\frac{1}{r} \frac{\partial}{\partial r}\left(r \int_{0}^{h(r, t)} u(r, z, t) \mathrm{d} z\right)+w(r, t)
$$

Injecting (10) into (12) and substituting $T_{e}(r)$ by (3) gives the evolution equation for the intrusion thickness $h$ :

$$
\begin{aligned}
\frac{\partial h}{\partial t}= & \frac{\rho_{m} g}{12 \mu} \frac{1}{r} \frac{\partial}{\partial r}\left(r h^{3} \frac{\partial h}{\partial r}\right)+\frac{\rho_{c} g \Psi T_{e}^{0}}{12 \mu} \frac{1}{r} \frac{\partial}{\partial r}\left(r h^{3} \frac{\partial \xi(r)}{\partial r}\right) \\
& +\frac{E T_{e}^{0^{3}}}{144 \mu\left(1-v^{2}\right)} \frac{1}{r} \frac{\partial}{\partial r}\left(r h^{3} \frac{\partial}{\partial r}\left(\nabla_{r}^{2}\left((1+\Psi \xi(r))^{3} \nabla_{r}^{2} h\right)\right)\right)+w(r, t)
\end{aligned}
$$

As expected, this evolution equation accounts for four different contributions. The first term on the right-hand side represents gravitational spreading of the intrusion; except for a constant arising from a no-slip boundary at the top of the flow, it is the same as for a gravity current [Huppert, 1982]. This term is negative and induces magma spreading. The second term is associated with the increase in lithostatic pressure at the crater wall zone and represents the lithostatic barrier the flow has to face when spreading below the crater wall zone; it is not present in the case of an overlying layer of constant thickness [Michaut, 2011]; this term is positive and opposes to the flow. The third term represents 
Table 2. Range of Values for the Model Parameters

\begin{tabular}{lcc} 
& Symbol & Range of Values \\
\hline Parameters & & \\
Depth of intrusion & $T_{e}$ & $0.5-5 \mathrm{~km}$ \\
Young's Modulus & $E$ & $1-10 \mathrm{GPa}$ \\
Poisson's ratio & $v$ & 0.25 \\
Gravity & $g$ & $1.62 \mathrm{~m} \mathrm{~s}^{-2}$ \\
Magma density & $\rho_{m}$ & $2800-3200 \mathrm{~kg} \mathrm{~m}^{-3}$ \\
Magma viscosity & $\mu$ & $1-10^{4} \mathrm{~Pa} \mathrm{~s}-$ \\
Feeder dyke width & $a$ & $10-100 \mathrm{~m}^{\circ}$ \\
Depth of the melt source & $Z_{c}$ & $200-500 \mathrm{~km}$ \\
Initial overpressure & $\Delta P$ & $1-20 \mathrm{MPa}^{-1}$ \\
Injection rate & $Q_{0}$ & $0.1-10^{8} \mathrm{~m}^{3} \mathrm{~s}^{-1}$ \\
Crust density & $\rho_{c}$ & $2500 \mathrm{~kg} \mathrm{~m}^{-3}$ \\
Crater depth & $D_{c}$ & $500-4000 \mathrm{~m}$ \\
Characteristic Scales & & \\
Height scale & & $1-35 \mathrm{~m}^{\circ}$ \\
Length scale & $H$ & $1-50 \mathrm{~km}$ \\
Time scale & $C$ & $10^{-3}-1 \mathrm{years}$ \\
Flexural wavelength & $\tau$ & $1-12 \mathrm{~km}$ \\
\hline
\end{tabular}

squeezing of the flow in response to the elastic deformation of the overlying layer. This term is negative and induces spreading in the case of an elastic layer of constant thickness [Michaut, 2011]. However, in the case of a layer that thickens with $r$, it can become positive and oppose to the flow if the thickening is rapid and important, i.e., at the crater wall zone. The last term represents the injection rate.

\subsection{Nondimensionalization}

Because the model depends on a large set of parameters (see Table 2), we nondimensionalize the flow equation (13) using the crater radius $C$, as defined in section 3.1 , as the characteristic length scale, a height scale $H$ and a time scale $\tau$ given by

$$
\begin{gathered}
H=\left(\frac{12 \mu Q_{0}}{\rho_{m} g \pi}\right)^{\frac{1}{4}}=a\left(\frac{3 \Delta P}{32 \rho_{m} g Z_{c}}\right)^{\frac{1}{4}} \\
\tau=\frac{\pi C^{2} H}{Q_{0}}=\left(\frac{12 \mu}{\rho_{m} g \pi}\right)^{\frac{1}{4}} \pi C^{2} Q_{0}^{-\frac{3}{4}}
\end{gathered}
$$

The height scale and the time scale are defined by equating the nondimensional group in front of the gravity current term to 1, i.e., $\left(\tau \rho_{m} g H^{3}\right) /\left(12 \mu C^{2}\right)=1$. The height scale $H$ is the characteristic height scale of a gravity current [Huppert, 1982]. $\tau$ is the time scale for a gravity current to fill in the crater; it mainly depends on the injection rate. In the case of an upper layer with constant thickness $T_{e}^{0}$, a characteristic length scale for the flow is the flexural parameter $\Lambda$, as defined by Turcotte et al. [1981], given by

$$
\Lambda=\left(\frac{E T_{e}^{0^{3}}}{12\left(1-v^{2}\right) \rho_{m} g}\right)^{\frac{1}{4}}
$$

It represents the wavelength over which the upper layer deforms elastically. However, in this problem, the crater radius $C$ imposes a horizontal length scale to the flow, as the lithostatic barrier the flow faces at the wall zone imposes a limitation to the intrusion spreading. The choice of $C$ as the length scale is then more relevant. Variables are then written as follows:

$$
r=C r^{*} h=H h^{*} t=\tau t^{*}
$$

where $r^{*}, h^{*}$, and $t^{*}$ are the dimensionless radius, thickness, and time. Substituting (17) into (13) and dropping the stars, we finally get the dimensionless equation for the flow:

$$
\begin{aligned}
\frac{\partial h}{\partial t}= & \frac{1}{r} \frac{\partial}{\partial r}\left(r h^{3} \frac{\partial h}{\partial r}\right)+\Xi \frac{1}{r} \frac{\partial}{\partial r}\left(r h^{3} \frac{\partial \xi(r)}{\partial r}\right) \\
& +\Theta \frac{1}{r} \frac{\partial}{\partial r}\left(r h^{3} \frac{\partial}{\partial r} \nabla_{r}^{2}\left((1+\Psi \xi(r))^{3} \nabla_{r}^{2} h\right)\right)+\frac{32}{\gamma^{2}}\left(\frac{1}{4}-\frac{r^{2}}{\gamma^{2}}\right)
\end{aligned}
$$

where $\xi(r)$ is also made dimensionless

$$
\xi(r)=\frac{1}{1+e^{-\frac{(r-1)}{\zeta}}}-\frac{1}{1+e^{\frac{1}{\zeta}}}
$$




\begin{tabular}{lccc}
\multicolumn{2}{l}{ Table 3. Dimensionless Numbers } & & \\
Complex Craters & Simple Craters \\
Symbol & Description & Range of Values & Range of Values \\
\hline$\gamma$ & Normalized source width & $10^{-4}$ to $10^{-2}$ & $10^{-4}$ to $10^{-2}$ \\
$\zeta$ & Normalized wall zone width & $0.05-0.13$ & 0.25 \\
$\Psi$ & Thickening term & $0.3-8$ & $0.2-4$ \\
$\Xi$ & Hydrostatic term & $20-1000$ & $1-500$ \\
$\Theta$ & Elastic term & $10^{-7}$ to 0.1 & $10^{-3}$ to 10 \\
$\Omega$ & Density ratio & 1.2 & 1.2 \\
$\Phi$ & Upper layer aspect ratio & 4500 & 1200 \\
$\sigma$ & Normalized pressure head & $0.6-100$ & $0.6-100$ \\
\hline \multicolumn{7}{c}{} & & $\Psi=\frac{D_{c}}{T_{e}^{0}}$ \\
& & & $\Theta=\left(\frac{\Lambda}{C}\right)^{4}$.
\end{tabular}

and where $\gamma, \zeta, \Xi, \Psi$, and $\Theta$ are five dimensionless numbers that control the dynamics of the flow.

$$
\begin{gathered}
\gamma=\frac{a}{C} \\
\zeta=\frac{D_{c}}{4 \alpha C} \\
\Xi=\left(\frac{\rho_{c} g D_{c}}{\rho_{m} g H}\right)
\end{gathered}
$$

The number $\gamma$ represents the dimensionless source width. The number $\zeta$ is the normalized crater wall zone width; its range of values has been discussed in section 3.1. The number $\Xi$ is the ratio between the lithostatic pressure increase at the crater wall zone and the hydrostatic pressure due to a magma column of thickness $H$. The number $\Psi$ is the dimensionless thickening of the upper elastic layer at the crater wall zone, as described in section 3.1. Finally, the number $\Theta$ is the dimensionless flexural wavelength of the upper layer elevated to the power 4; it quantifies the length scale over which the elastic deformation is effective relative to the crater radius. Additionally, we define a last dimensionless number $\Omega$

$$
\Omega=\frac{\rho_{m}}{\rho_{c}}
$$

which is the ratio between the magma and crust density. The value of $\Omega$ is set equal to 1.2 . The dimensionless topography is then given by

$$
T_{p}(r)=\Xi \Omega \xi(r)
$$

To obtain the crater floor appearance in the different figures, we add to the dimensionless expression of the topography, the dimensionless thickness $h(r)$ of the intrusion.

\subsection{Range of Values for the Dimensionless Numbers}

For a conduit diameter varying between 10 and $100 \mathrm{~m}$ and a crater radius between 10 and $50 \mathrm{~km}$, the normalized source width $\gamma$ (20) varies between $10^{-5}$ and $10^{-2}$ (Table 3). Its variation does not significantly influence the results and in particular, does not play on the shape of the intrusion [Michaut, 2011]; it is fixed at a value of 0.02 in this study.

For a Young's modulus between 1 and $10 \mathrm{GPa}$, a gravity of $1.62 \mathrm{~m} \mathrm{~s}^{-2}$, a magma density $\rho_{m}$ between 2800 and $3200 \mathrm{~kg} \mathrm{~m}^{-3}$, and an elastic thickness $T_{e}^{0}$ ranging from 0.5 to $5 \mathrm{~km}$, the flexural wavelength of the upper layer $\Lambda$, given by (16), varies between 1 and $12 \mathrm{~km}$ (Table 2). Accordingly, for complex craters between 20 and $100 \mathrm{~km}$ in diameter, the length scale $C$ is between $6 \mathrm{~km}$ and $40 \mathrm{~km}$ and the number $\Theta$ takes values between $10^{-7}$ and 1 (Table 3). For simple craters between 10 and $20 \mathrm{~km}$ in diameter, the number $\Theta$ is between $10^{-3}$ and 10 .

For complex craters, the crater depth ranges from 2 to $4 \mathrm{~km}$ and the number $\Psi$ varies between 0.3 and 8 for intrusions between 0.5 and $5 \mathrm{~km}$ depth. For simple craters 1 to $2 \mathrm{~km}$ deep, this number is between 0.2 and 4 (Table 3). Additionally, the characteristic thickness $H$ for a gravity current of basalts is between 0.1 and $20 \mathrm{~m}$ and hence, the number $\Xi$ takes values between 1 and 500 for simple craters and 20 and 1000 for complex craters. Furthermore, we use $\zeta=0.25$ for simple craters and $\zeta=0.13$ for complex craters (see section 3.1).

In summary, we investigate the influence of four dimensionless numbers on the flow dynamics: the number $\zeta$, through the effect of two end-member topographies (see section 3.1), and the numbers $\Xi, \Theta$, and $\Psi$. 


\section{Results}

Equation (18) is solved numerically using a fully implicit finite-volume method detailed in Appendix A. In all solutions, we compute the mass conservation as a test for the accuracy of the convergence. We first examine the case of an intrusion below a strengthless overlying layer whose thickness varies radially according to (3). We then consider the case of an intrusion that lies beneath an elastic layer whose thickness is also given by (3).

\subsection{Intrusion Below a Brecciated Zone With no Elastic Strength (Effect of $\Xi$ )}

If the layer overlying the intrusion is highly fractured and brecciated, it is strengthless and $\Theta=0$. In that case, we solve

$$
\frac{\partial h}{\partial t}=\frac{1}{r} \frac{\partial}{\partial r}\left(r h^{3} \frac{\partial h}{\partial r}\right)+\Xi \frac{1}{r} \frac{\partial}{\partial r}\left(r h^{3} \frac{\partial \xi(r)}{\partial r}\right)+\frac{32}{\gamma^{2}}\left(\frac{1}{4}-\frac{r^{2}}{\gamma^{2}}\right)
$$

which corresponds to (18) without the elastic term.

We first consider an intrusion below a complex crater characterized by a topography given by (19) using $\zeta=$ 0.13. In that case, at the crater wall, the overlying layer thickens and the flow faces an increase in lithostatic pressure which becomes more important as $\Xi$ increases. To examine the effect of the lithostatic pressure increase at the wall zone on the intrusion spreading, we consider different values of $\Xi=20,50$, and 200 in (27).

Up to $t \sim 1$, the flow spreads as a classical gravity current as the lithostatic term (i.e., second term on the right-hand side of (27)) is negligible. In this regime, the flow thickness goes toward a constant, noted $H_{g}$, of order 1 (which depends on the source width [Michaut and Bercovici, 2009]) while the radius evolves as $t^{1 / 2}$ as predicted by the gravity current theory [Huppert, 1982] (Figures $4 \mathrm{a}$ and $4 \mathrm{~b}$ ). The intrusion shows a characteristic flat top profile with a steep front (Figure 4c). When the intrusion reaches the wall zone, at $t \sim 1$, the lithostatic pressure increase prevents the intrusion from spreading laterally. The second term on the right of (27) is positive, it increases with $\Xi$ and causes the thickening of the intrusion (Figures 4a and 4c). In this thickening regime, the edge radius remains close to a constant (Figure 4b). At the surface, the thickening of the flat top intrusion results in an important piston-like uplift of the crater floor. However, no moat is formed adjacent to the wall zone (Figure 4c).

In contrast to complex craters, the topography of simple craters, and hence the lithostatic pressure, gradually increases from the center to the exterior of the crater wall zone. We use $\zeta=0.25$ and $\Xi=5$ in (27). In that case, there is no gravity current phase; the gradual lithostatic pressure increase slows down the spreading from the beginning and induces a gradual thickening phase (Figures $4 d, 4 e$, and $4 f$ ). The intrusion shape almost accommodates the topography (Figure 4f). Indeed, because the overlying layer is less dense than the magma, the crater floor appears slightly concave up, the concavity increasing with the density difference between the overlying layer and the magma, i.e., with $\Omega$ (Figure $4 \mathrm{f}$ ).

For both types of craters, the phase of thickening ends when the hydrostatic pressure due to the weight of the magma equals the lithostatic pressure outside of the wall zone, i.e., $h_{0} \sim \Xi$. When $h_{0}$ becomes larger than $\Xi$, the gravity current term compensates for the lithostatic term in (27) and the intrusion can flow down the wall zone and return in a gravity current regime (Figures $4 a, 4 b, 4 d$, and $4 \mathrm{e}$ ). In the gravity current regime, the flow evolves toward a constant value equal to $\Xi+H_{g}$. Thus, for a constant injection rate, the number $\Xi$ mostly controls the final intrusion thickness and hence the shallowing of the crater floor. However, the injection rate might decrease as the intrusion grows and its weight compensates for the overpressure at the origin of magma ascent. This effect should control the final shallowing of the crater floor and is discussed in section 4.5 .

\subsection{Intrusion Below an Elastic Layer of Constant Thickness} In the previous section, we considered that the intrusion stalls beneath a highly fractured and brecciated zone with no strength. However, an impact melt unit might be present on top of the brecciated lens. Furthermore, the intrusion might also emplace deeper than the brecciated lens. Both cases imply the deformation of an upper coherent elastic layer during the spreading of the intrusion. In Michaut [2011], the model was developed for an upper elastic layer of constant thickness in a 2-D geometry and the behavior of the flow was predicted for an axisymmetric geometry. Here we first show the results for an elastic layer with a 


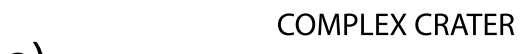

a)

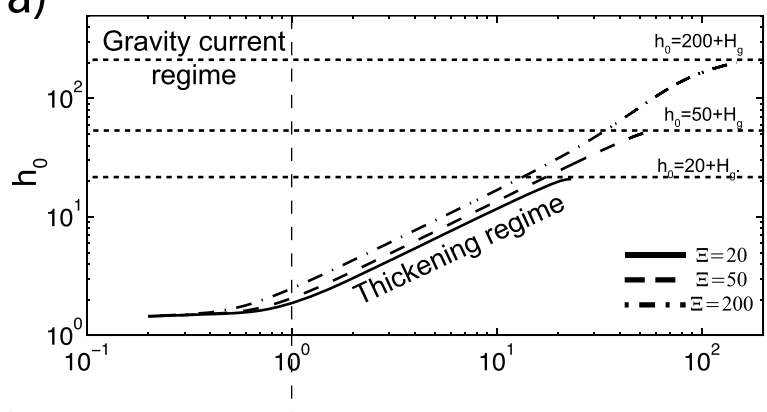

b)

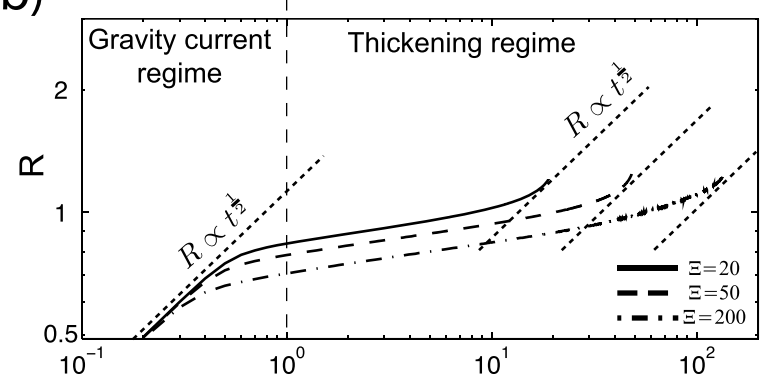

C)

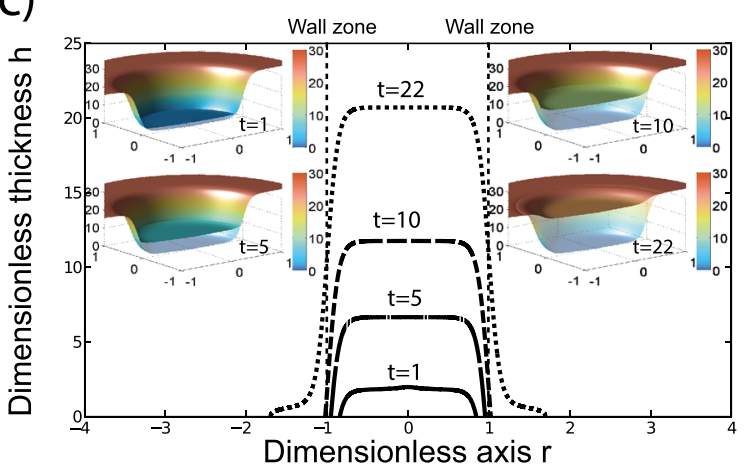

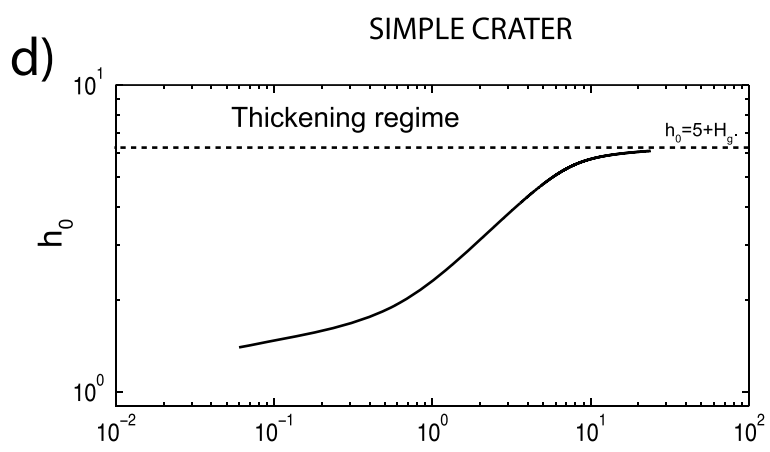

e)

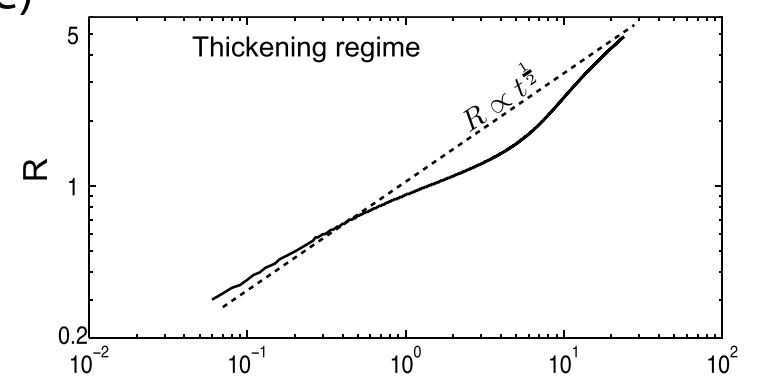

f)

Dimensionless time $\mathrm{t}$

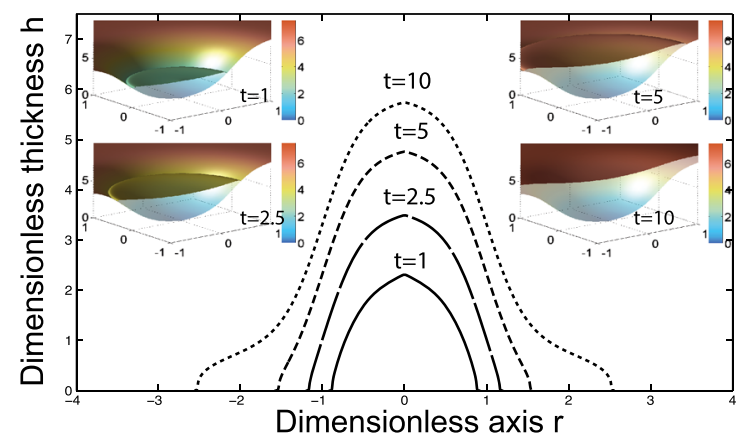

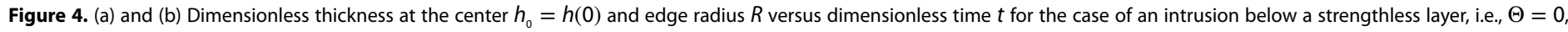

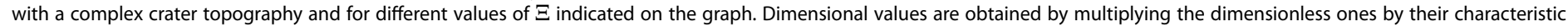

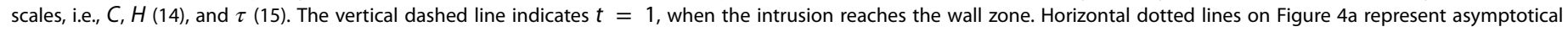

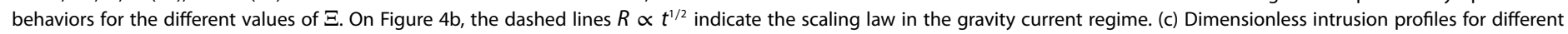

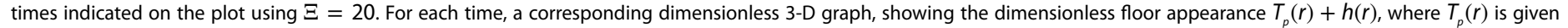

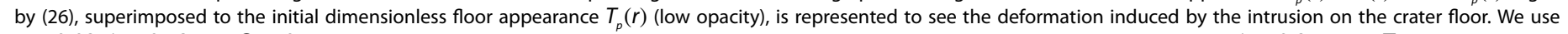
$\gamma=0.02, \zeta=0.13$, and $\Theta=0$. (d, e, and f) Same plots but for an intrusion below a strengthless layer with a simple crater topography, i.e., $\zeta=0.25$, using $\Xi=5$.

constant thickness in an axisymmetric geometry and verify the predictions of Michaut [2011]. In the next sections, we examine the case of an upper elastic layer with a crater-like topography and a thickness that varies with the radius according to (3).

We first solve equation (18) using $\Theta=1, \Xi=0$, and $\Psi=0$, i.e., $T_{e}(r)=T_{e}^{0}$ to model the case of a magma that flows beneath an upper elastic layer of constant thickness. Equation (18) becomes

$$
\frac{\partial h}{\partial t}=\frac{1}{r} \frac{\partial}{\partial r}\left(r h^{3} \frac{\partial h}{\partial r}\right)+\frac{1}{r} \frac{\partial}{\partial r}\left(r h^{3} \frac{\partial}{\partial r}\left(\nabla_{r}^{4} h\right)\right)+\frac{32}{\gamma^{2}}\left(\frac{1}{4}-\frac{r^{2}}{\gamma^{2}}\right)
$$

In this case, there is no limitation to the flow as both the gravity current term and the elastic term, i.e., the first and second terms on the right side of (28), cause spreading. As expected, the intrusion dynamics follows two different spreading regimes [Michaut, 2011; Michaut et al., 2013; Bunger and Cruden, 2011]. During the first phase, the thickening is important and the intrusion shows a bell-shaped profile (Figures 5a and 5c). As shown by Michaut [2011], the flow dynamics is controlled by the elastic deformation of the overlying layer up to a radius $R=4 \Lambda$. In this regime, the elastic pressure is dominant over the pressure due to 

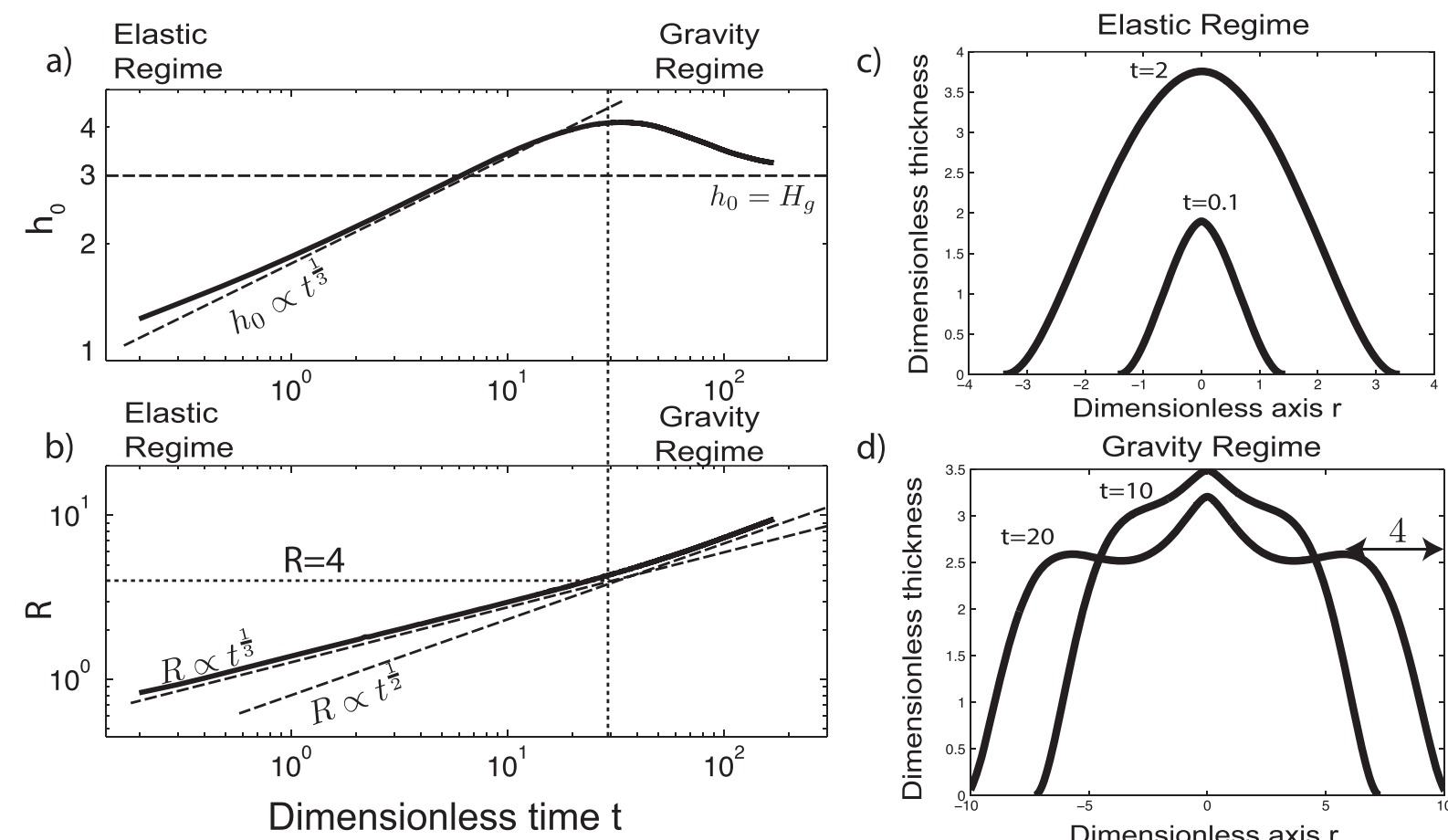

d)

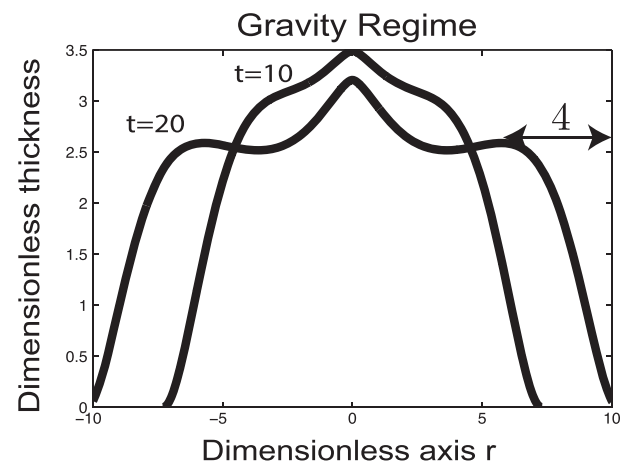

Figure 5. (a) and (b) Dimensionless thickness at the center $h_{0}=h(0)$ and edge radius $R$ versus dimensionless time $t$ for the case of an intrusion below a constant thickness elastic layer. For $R<4$, the flow dynamics is controlled by the elastic deformation of the overlying layer, while for an $R>4$, it is controlled by its own weight. In Figure 5 a, the dashed lines correspond to $h_{0} \propto t^{1 / 3}$ and $h_{0}=H_{g}$ which provide scaling laws for the elastic and gravity current regimes. In Figure 5b, the dashed lines $R \propto t^{1 / 3}$ and $R \propto t^{1 / 2}$ represent scaling laws for the elastic and gravity current regimes [Michaut, 2011]. The vertical dashed line indicates the time when the intrusion reaches a radius equal to 4 . The intrusion shape is shown at different times in the elastic regime (c) and in the gravity current regime (d). We use $\gamma=0.02, \Xi=0, \Psi=0$, and $\Theta=1$.

magma weight. In the magma, the pressure is constant below the elastic layer, except at the tip [Bunger and Cruden, 2011; Michaut, 2011], which explains the bell-shaped profile of the intrusion (Figure 5c). Indeed, for a constant pressure $P_{d}$ in the magma, $P_{e}(r)=D \nabla_{r}^{4} h=P_{d}$ implies

$$
h(r)=\frac{P_{d} R^{4}}{64 D}\left(1-\left(\frac{r}{R}\right)^{2}\right)^{2}
$$

where $D$ is the flexural rigidity of the upper layer of constant thickness as defined by (9). Results show that the thickness and radius evolve with time with an exponent close to 1/3, as predicted by Michaut [2011] when neglecting the gravity current term and considering a constant injection rate (Figures $5 \mathrm{a}$ and $5 \mathrm{~b}$ ). Beyond $R=4 \Lambda$, the size of the intrusion becomes much larger than the flexural parameter and the gravitational term becomes prevalent in the dynamics. The intrusion thus transitions to a gravity current regime characterized by a flat-top profile (Figure $5 \mathrm{~d}$ ). In this regime, the flow thickness goes toward a constant $H_{g^{\prime}}$ while the radius follows $t^{1 / 2}$ as predicted by the gravity current theory [Huppert, 1982] (Figures 5a and 5b). The elastic term is negligible when considering the dynamics of the whole flow in this regime. However, the overlying layer is unable to accommodate the steep front of a typical gravity current flow [Huppert, 1982] and the intrusion develops a small scale bent edge whose width is equal to $4 \Lambda$ (Figure $5 d$ ).

\subsection{Spreading Beneath a Complex Crater}

In this section, we consider an intrusion below an elastic overlying layer with a complex crater topography, hence, we use $\zeta=0.13$ in (19). We first examine the effect of varying the dimensionless flexural parameter, i.e., the dimensionless number $\Theta$ and then examine the influence of the thickening at the wall by varying the number $\Psi$.

\subsubsection{Effect of the Dimensionless Flexural Parameter (Effect of $\Theta$ )}

We consider equation (18) for which we set $\Xi=20$ and $\Psi=0.3$ and consider two different values of the dimensionless flexural parameter $\Theta=10^{-2}$ and $\Theta=10^{-5}$. Up to $t \sim 1$, the central flat floor of the crater acts as a constant thickness upper layer and the lithostatic term (second term on the right-hand side of (18)) is negligible. 

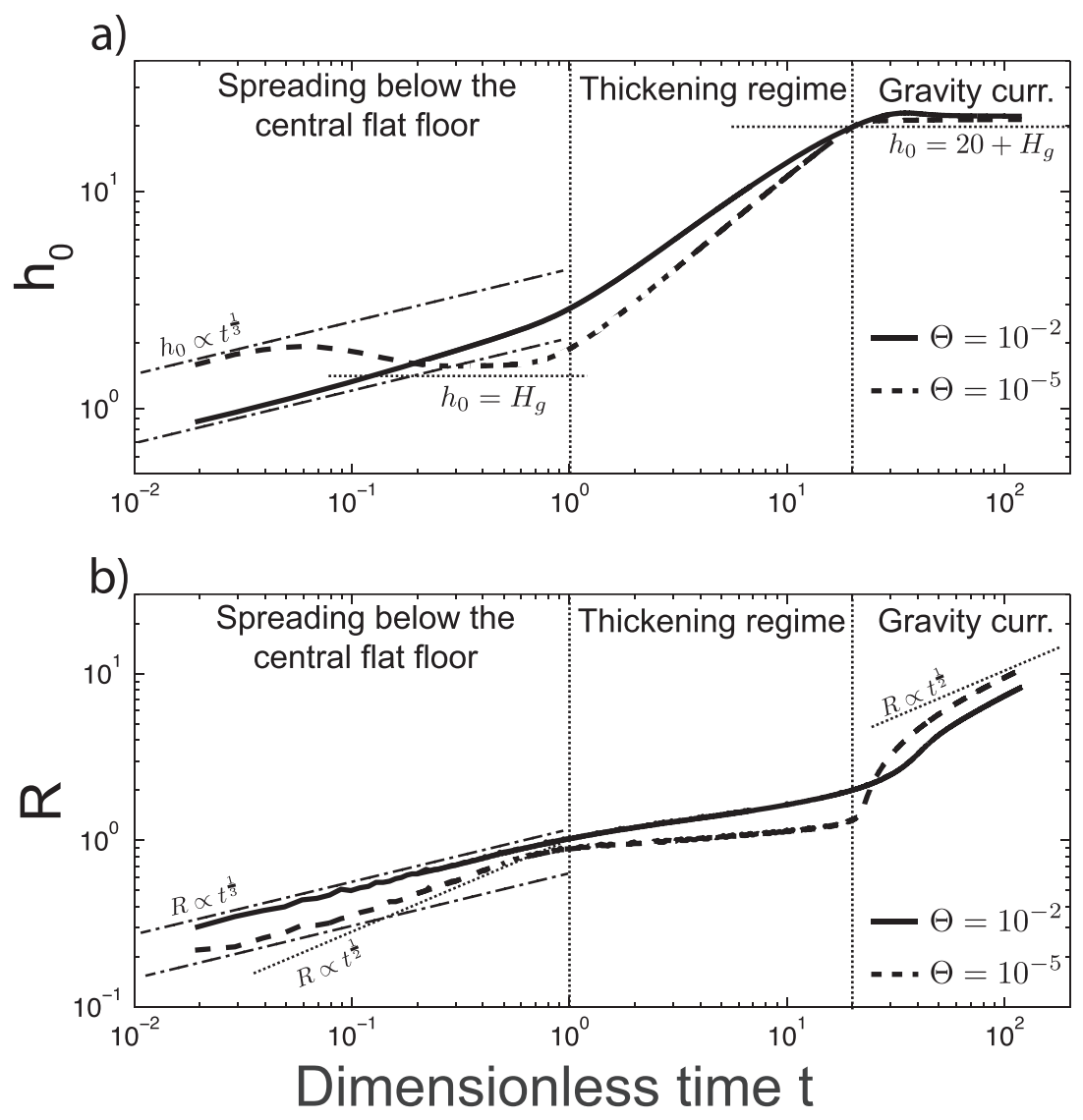

Figure 6. Dimensionless thickness at the center $h_{0}=h(0)$ and edge radius $R$ versus dimensionless time $t$ for the case of an intrusion spreading below an overlying elastic layer with a topography characteristic of a complex crater, i.e., $\zeta=0.13$ and using a constant injection rate. Vertical dotted lines: dimensionless times when the intrusion enters and leaves the thickening regime. Thick solid lines, $\Theta=10^{-2}$, thick dashed lines, $\Theta=10^{-5}$. Scaling laws are shown in dash-dotted lines for the elastic regime $\left(R \propto t^{1 / 3}, h_{0} \propto t^{1 / 3}\right)$ and in dotted lines for the gravity current regime $\left(R \propto t^{1 / 2}, h_{0} \rightarrow H_{g}, h_{0} \rightarrow \Xi+H_{g}\right)$. We use, $\gamma=0.02, \zeta=0.13, \Xi=20$ and $\Psi=0.3$.

For $\Theta=10^{-2}$, the flexural wavelength is close to the crater size, i.e., $4 \Lambda \sim C$. The intrusion spreads in an elastic regime below the crater flat floor, the dimensionless radius and thickness evolutions closely parallel $t^{1 / 3}$ (Figure 6), and the intrusion shows a characteristic bell-shaped profile (Figure 7a). At $t \sim 1$, the second term on the right-hand side of (18), representing the lithostatic pressure increase at the crater wall zone, becomes important and opposes to the flow. The intrusion enters a thickening dominated regime, where the thickness increases significantly and the radius remains close to constant (Figures 6 and 7a), while it has not yet transitioned into a gravity current regime and still shows a bell shape. The thickening of the bell-shaped intrusion results in a convex uplift of the crater floor (Figure 7a).

For $\Theta=10^{-5}$, the intrusion first spreads in an elastic regime and the dimensionless radius and thickness evolve as $t^{1 / 3}$ (Figure 6). However, the flexural wavelength is much smaller than the crater size in this case (i.e., $4 \Lambda<<C$ ). The flow transitions to a gravity current regime far in front of the wall zone, at $t=0.06$, which is evident as, beyond this time, the dimensionless radius evolves as $t^{1 / 2}$ while the dimensionless thickness goes toward a constant (Figures $6 \mathrm{a}$ and $6 \mathrm{~b}$ ). In consequence, the intrusion shows a flat-top profile and a small scale bent edge while reaching the crater wall zone (Figure $7 \mathrm{~b}$ ). At $t \sim 1$, the lithostatic pressure increase prevents the intrusion from spreading radially and the intrusion thickens. The thickening of the flat-top intrusion results in a piston-like uplift of the central part of the crater floor (Figures 6a and 7b) as in the previous case of a gravity current below a strengthless overlying layer (Figure 4c). However, the bending of the upper layer above the intrusion at its edge leads to the formation of a circular moat, adjacent to the wall zone, whose extent corresponds to the size of the intrusion edge and is $\sim 4 \Lambda$ (Figure $7 \mathrm{~b}$ ).

The thickening rate of the intrusion, as it reaches the wall zone, depends on its spreading regime (Figures 6a and $6 b$ ) and hence on the value of $\Theta$. For larger values of $\Theta$, the elastic pressure that squeezes the flow is 

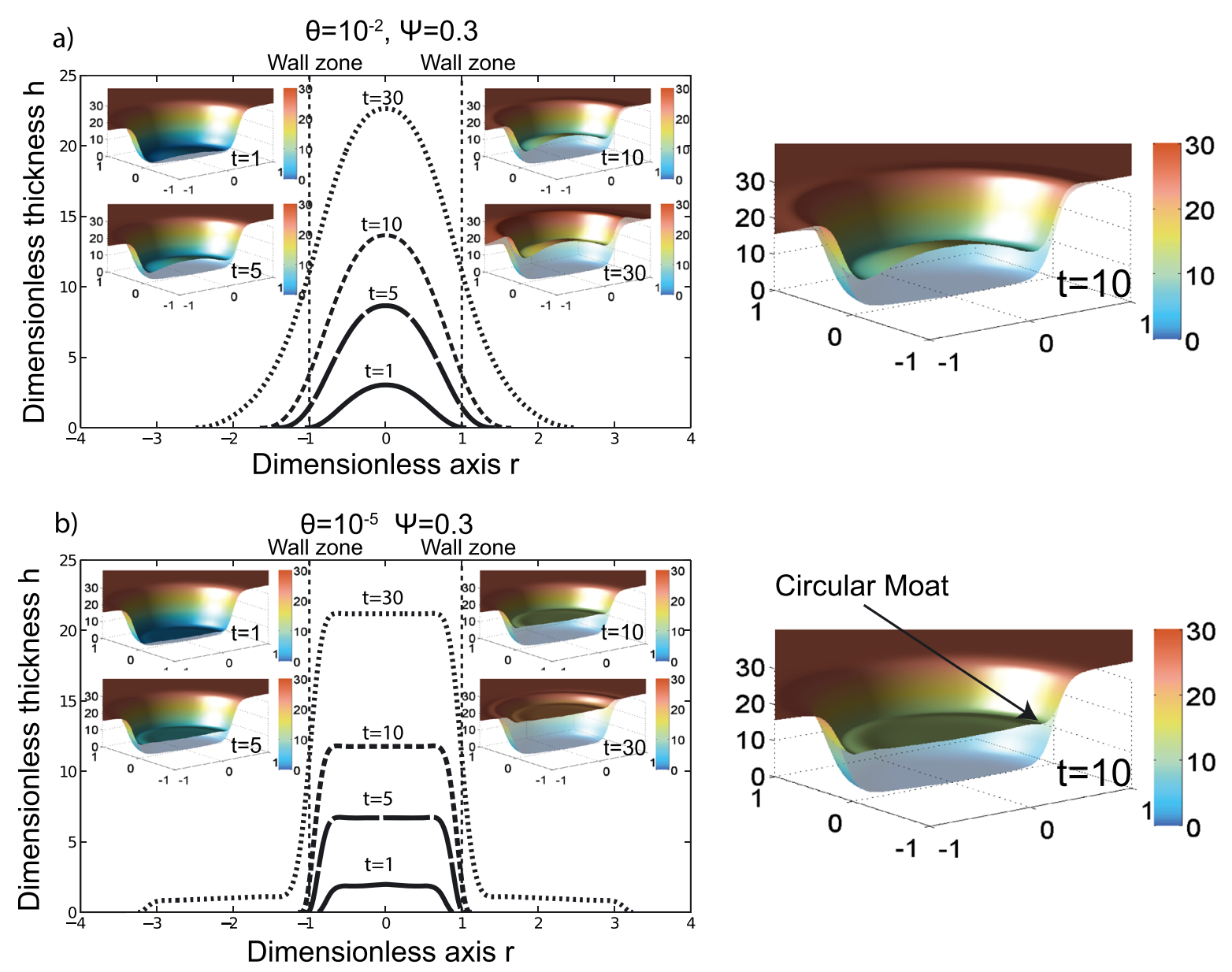

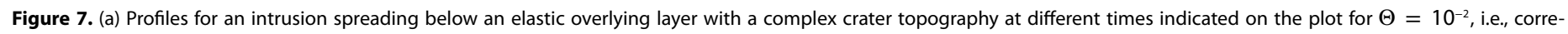

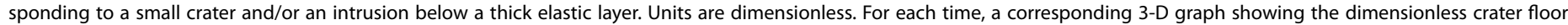

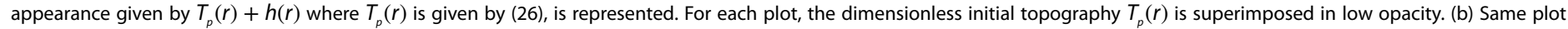
but for a large crater and/or a shallow intrusion, i.e., $\Theta=10^{-5}$. Here we use $\gamma=0.02, \zeta=0.13, \Xi=20$, and $\Psi=0.3$.

more important and it is easier for the magma to overcome the lithostatic barrier and spread below the wall zone. In any case, when the sum of the pressures due to elastic bending and to magma weight compensates for the hydrostatic pressure due to the crater wall zone weight, the flow passes the wall zone. For both values of $\Theta$, as it passes down the wall zone, the intrusion is in a gravity current regime as $R$ is or becomes larger than $4 \Lambda$ : the radius evolves as $t^{1 / 2}$ and the thickness evolves toward the final value of $\Xi+H_{g}$ (Figures 6a and $6 b)$.

\subsubsection{Effect of the Local Increase in the Flexural Parameter at the Wall Zone (Effect of $\Psi$ )}

Due to the elastic thickness increase at the crater wall zone, the flexural wavelength of the overlying elastic layer increases from $\Lambda$ at the crater center to a value equal to

$$
\Lambda_{b}=\left(\frac{E\left(T_{e}^{0}+D_{c}\right)^{3}}{12\left(1-v^{2}\right) \rho_{m} g}\right)^{\frac{1}{4}}=\Lambda(1+\Psi)^{\frac{3}{4}}
$$

beyond the wall zone. To understand the effect of the local increase in flexural wavelength at the wall zone, we consider a constant value of the number $\Xi=20$ and increasing values of the number $\Psi=0.3,3$, and 6 for the two different values of the number $\Theta=10^{-2}$ and $\Theta=10^{-5}$ used previously and representing two possible end-member floor deformations.

For $\Theta=10^{-2}$, the elastic term is dominant over the gravity current term as the flow arrives to the wall zone and the effect of the local increase in flexural wavelength of the overlying layer is to increase the thickening rate with time (Figure 8a). The increase in flexural wavelength enhances the barrier effect of the lithostatic pressure increase at the crater wall zone, and in response, the flow thickens rapidly in the center, where 
a)
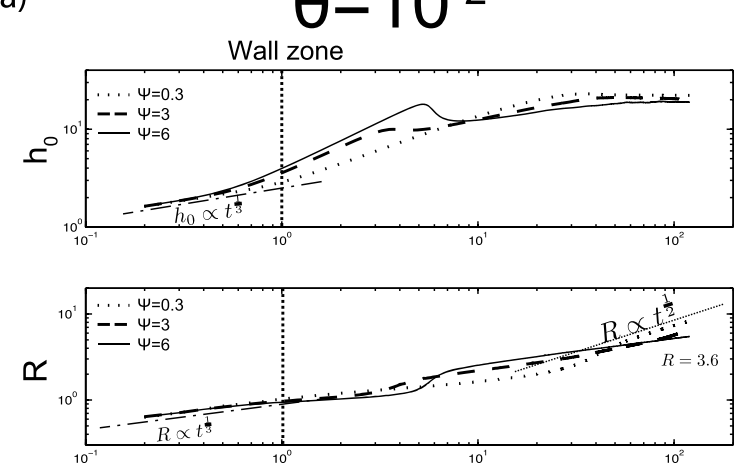

c)

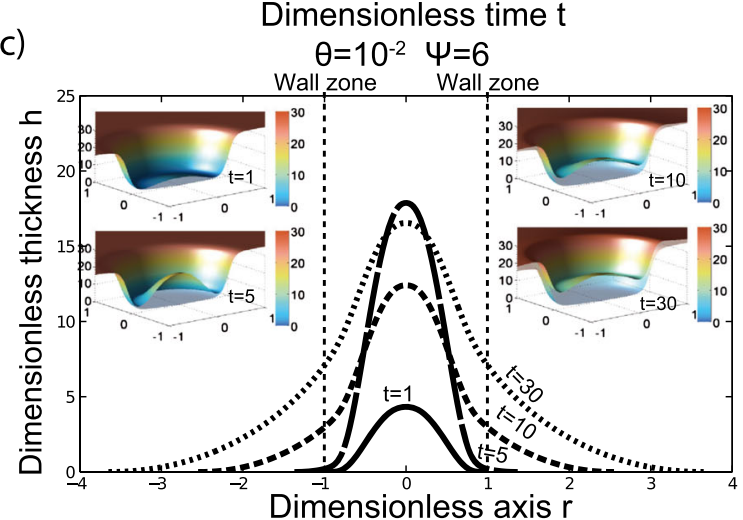

e)

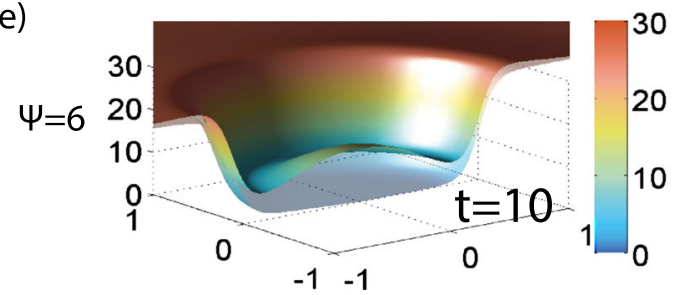

b)

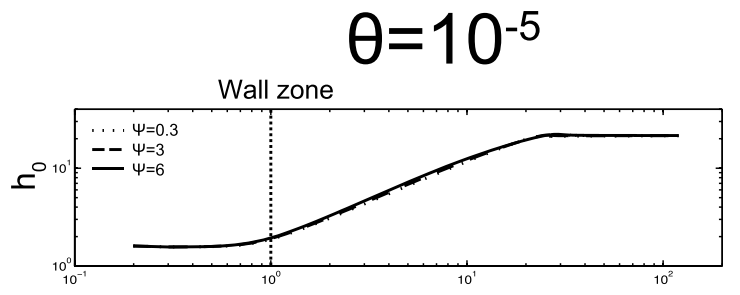

d)

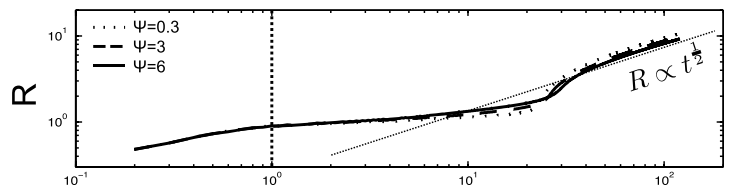

Dimensionless time $\mathrm{t}$

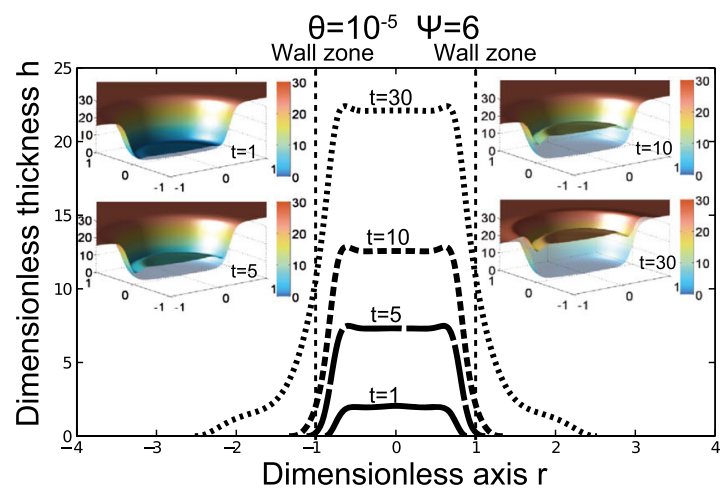

f)

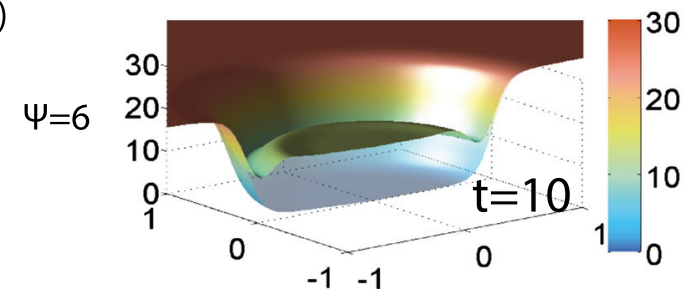

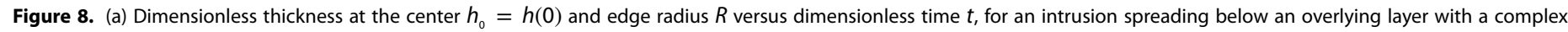

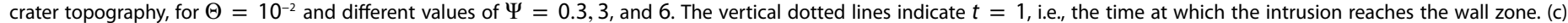

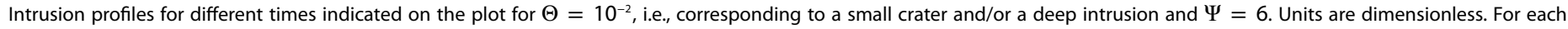

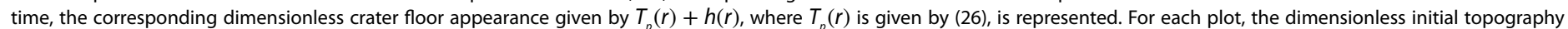

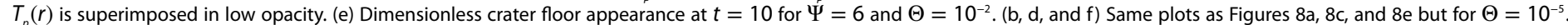
We use $\gamma=0.02, \zeta=0.13$, and $\Xi=20$.

the bending is easier (Figures $8 \mathrm{a}$ and $8 \mathrm{c}$ ). The elastic pressure increases with the bending and after a time that increases with $\Psi$, it becomes important enough to compensate for the lithostatic pressure increase at the wall zone and induces a squeezing of the intrusion below the wall zone. For large values of $\Psi$, the local increase in flexural wavelength is important at the wall zone and forces the flow to extend and deform the overlying layer over a much larger distance, beyond the crater, than for smaller values of $\Psi$ (compare $\Psi=0.3$ and 6 on Figure 8a). As a result, for large values of $\Psi$, the intrusion profile relaxes, the thickness at the center collapses (Figures $8 \mathrm{a}$ and $8 \mathrm{c}$ ), and the radius increases quickly. After the relaxation of the profile, the intrusion is still in an elastic regime but characterized by a flexural parameter $\Lambda_{b}$. Once the dimensionless radius reaches $r=4 \Lambda_{b} / C=4 \Lambda(1+\Psi)^{3 / 4} / C=4 \Theta^{1 / 4}(1+\Psi)^{3 / 4}$, the intrusion transitions to a gravity current regime where the thickness evolves toward a constant and the radius grows as $t^{1 / 2}$ (Figure $8 \mathrm{a}$ ). For instance, for $\Psi=3$, the transition to a gravity current regime occurs when $R=4 \Theta^{1 / 4}(1+\Psi)^{3 / 4}=3.6$ (Figure 8a).

For $\Theta=10^{-5}$, the gravity current term is dominant when the intrusion reaches the wall zone and the elastic term only controls the shape of the front (Figures $8 \mathrm{~b}$ and $8 \mathrm{~d}$ ). And hence, the thickening rate only increases by a few percent between $\Psi=0.3$ and $\Psi=6$, while it increases by a factor of 3 in the case of $\Theta=10^{-2}$ (Figures $8 \mathrm{a}$ and $8 \mathrm{~b}$ ). Nevertheless, the local increase in the flexural wavelength below the wall zone tends 
to enlarge the size of the intrusion edge and to result in a deeper circular moat at the surface (Figures $8 \mathrm{~d}$ and $8 f)$.

\subsection{Spreading Beneath a Simple Crater}

In comparison to complex craters, the lithostatic pressure and the flexural wavelength of the upper layer gradually increase from the center to the exterior of the crater wall zone. Given the appropriate range of values for the number $\Theta=10^{-3}-10$, as discussed in section 3.4, the flexural parameter is between 0.2 and 1 times the crater radius $C$. The bending of the upper layer thus controls the intrusion shape and dynamics during the whole flow, and the intrusion always shows a bell-shaped geometry (Figure 9). To represent the case of an intrusion below a bowl-shaped crater, we use $\zeta=0.25, \Xi=20$, and $\Theta=0.1$, and we study the effect of the thickening at the wall zone by using different values of $\Psi=0.1$ and $\Psi=4$.

For $\Psi=0.1$, the thickening at the wall zone $\Psi \xi(r)$ is negligible and the elastic term is similar to that of a constant thickness layer. However, the lithostatic pressure increase slows down the spreading and the evolution of the radius with time is slower than for an elastic regime below a constant thickness layer. The intrusion shows a bell-shaped profile with an important curvature because the weight of the lithostatic barrier constrains the intrusion edge (Figure 9a). For $\Psi=4$, the local flexural wavelength rapidly rises up to $\Lambda_{b}=\Lambda(1+\Psi)^{3 / 4}$. As a consequence, the intrusion squeezes more rapidly and thickens more slowly than for smaller values of $\Psi$ (Figure $9 \mathrm{~b}$ ). For a larger value of $\Psi$, the deformation occurs over a larger wavelength and the shallowing is smaller at a given time. However, the overall deformation the surface is very similar in both cases: the crater floor appears convex and shows an important curvature which overlaps the initial bowl shape of the crater (Figures 9a and 9b).

\subsection{Effect of a Variable Injection Rate}

So far, we have assumed a constant injection rate; and hence the intrusion grows indefinitely in volume and the flow passes the crater wall zone. However, the driving pressure in the feeder conduit should decrease as the intrusion grows during the thickening phase. In particular, the increase in hydrostatic pressure due to magma weight and in elastic pressure due to bending above the dyke might finally compensate for the initial overpressure $\Delta P$ in the magma at the origin of the flow. As the increase in lithostatic pressure at the crater wall zone prevents the intrusion from spreading laterally, the intrusion might reach a steady state profile such that no more magma can intrude. New expressions for the vertical injection velocity and injection rate, taking into account the increase in pressure in the magma at the top of the feeder dyke, are given by

$$
w(r, t)=\frac{32}{\gamma^{2}}\left(1-\frac{1}{\sigma}\left(h_{0}+P_{e, r=0}\right)\right)\left(\frac{1}{4}-\frac{r^{2}}{\gamma^{2}}\right)
$$

and

$$
Q_{0}=\left(1-\frac{1}{\sigma}\left(h_{0}+P_{e, r=0}\right)\right)
$$

where

$$
\sigma=\frac{\Delta P}{\rho_{m} g H}
$$

and

$$
P_{e, r=0}=\left.\Theta \nabla_{r}^{2}\left((1+\Psi \xi(r))^{3} \nabla_{r}^{2} h\right)\right|_{r=0}
$$

The dimensionless number $\sigma$ represents the normalized pressure head, $P_{e, r=0}$ is the dimensionless elastic pressure at the center, and $Q_{0}$ the dimensionless total injection rate. As discussed in Michaut [2011], the elastic pressure $P_{e, r=0}$ required to bend the upper layer tends to infinity as $R \rightarrow 0$, but rapidly decreases with the radius as $P_{e, r=0}$ scales with $h_{0} / R^{4}$ (see (8)). However, here we assume a large aspect ratio for the intrusion (lubrication assumption), and this initial transient regime, where the flow is very narrow and $P_{e, r=0}$ is very large, is thus not representative of the initiation phase of the intrusion. Hence, to investigate the effect of the elastic pressure on the injection rate, we neglect this initial transient phase.

Given that the deformations are limited to craters, it is possible that excavation of material during the impact has controlled the intrusion of magma. In that case, the driving pressure $\Delta P$ at the origin of the flow should be lower or equal to the weight of crust that has been removed by cratering, i.e., $\Delta P \leq \rho_{c} g D_{c}$, which implies 

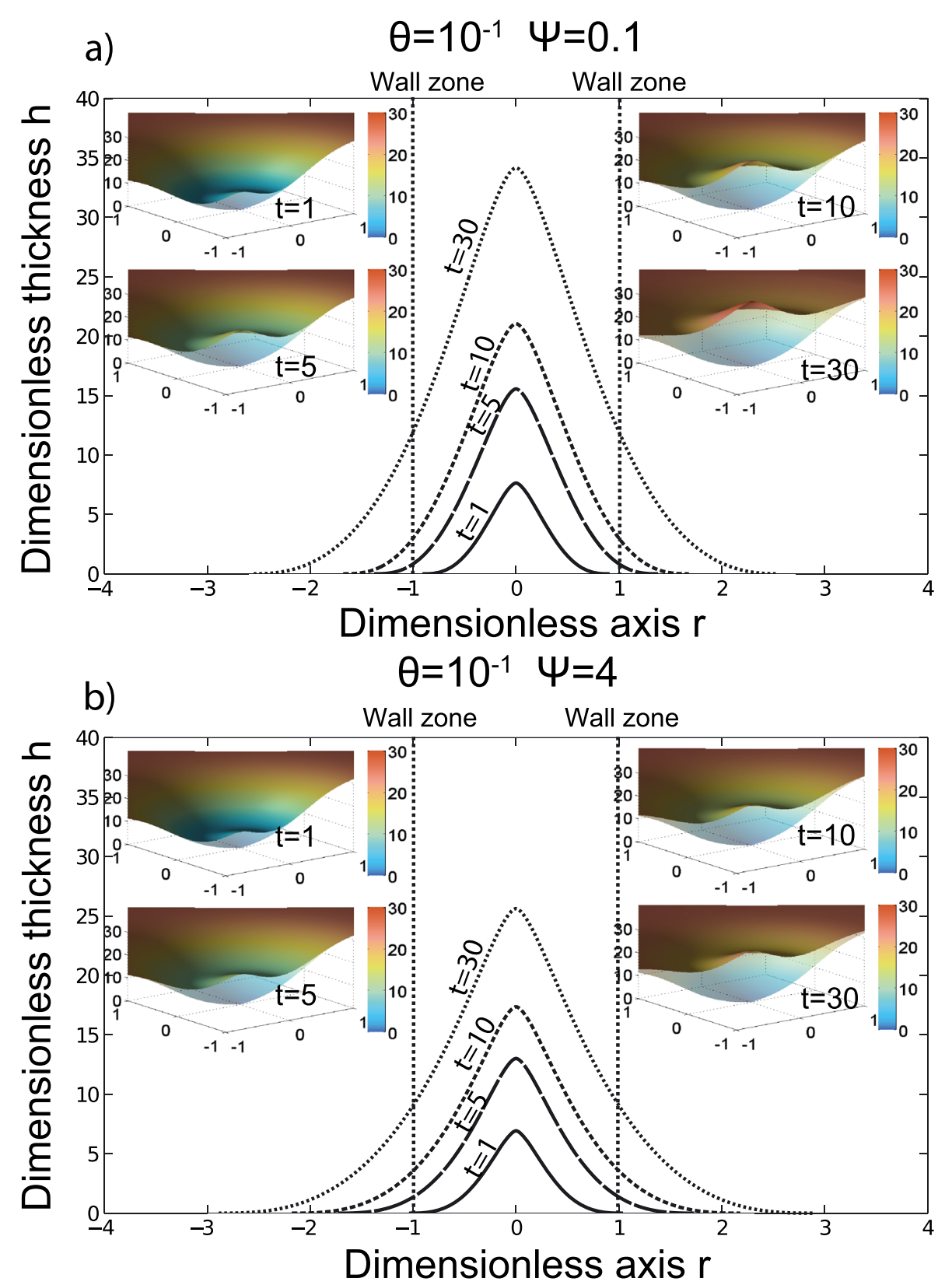

Figure 9. (a) Dimensionless profiles of an intrusion spreading below an overlying elastic layer with a simple crater topography, i.e., $\zeta=0.25$, for different dimensionless times indicated on the plot for $\Theta=10^{-1}$ and $\Psi=0.1$. For each time, a corresponding 3-D graph, showing the dimensionless crater floor appearance given by $T_{p}(r)+h(r)$, where $T_{p}(r)$ is given by (26), is represented. For each plot, the initial topography $T_{p}(r)$ is superimposed in low opacity. (b) Same plot but for $\Psi=4$. We use, $\gamma=0.02$ and $\Xi=20$.

$\sigma<\Xi$ (see (22) and (33)). Hence, given the range of values for the parameters (Table 2), the value of $\sigma$ varies between 0.6 and 100 (Table 3). We consider a complex crater with $\zeta=0.13$, and we set $\Xi=50, \Psi=1$, $\sigma=22$, and two values of $\Theta$ equal to $10^{-2}$ and $10^{-5}$, to consider the effect of a variable injection rate.

For $\Theta=10^{-2}$, the elastic pressure governs the spreading of the intrusion below the crater flat floor; as $P_{e, r=0}$ scales with $h_{0} / R^{4}, P_{e, r=0}$ decreases as the flow spreads and its radius increases (Figure 10a) inducing an increase in the total injection rate $Q_{0}$ (32). Then, as the intrusion thickens, both the pressure due to magma weight and the elastic pressure increases at the center, in response (Figure 10a). The total injection rate decreases, and the system reaches a steady state shape when $Q_{0} \rightarrow 0$, i.e., when the initial overpressure is compensated by the sum of the elastic pressure and of the pressure due to magma weight (Figures 10a and 10b). 
a)

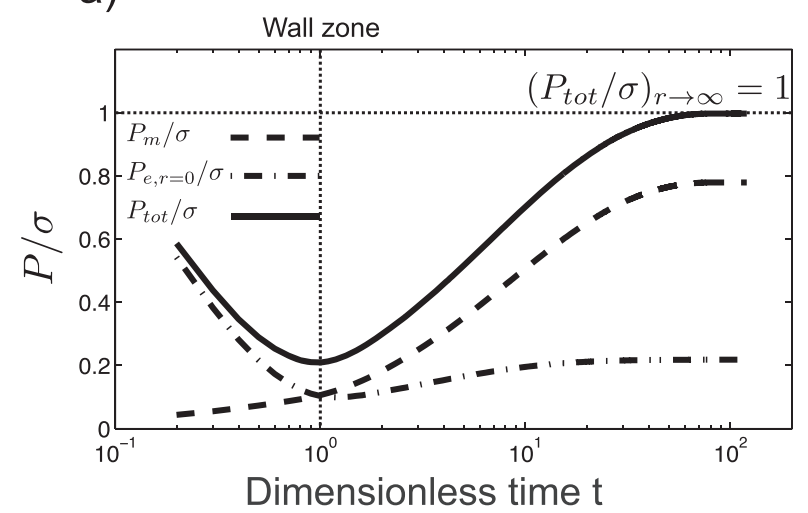

c)

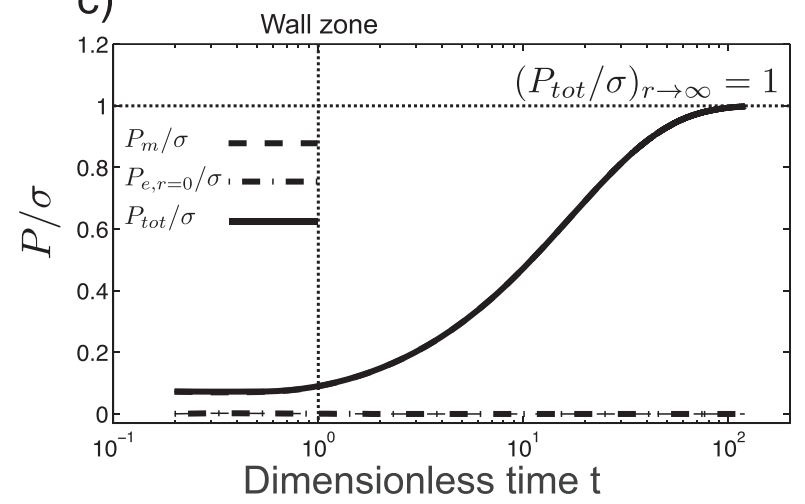

b)

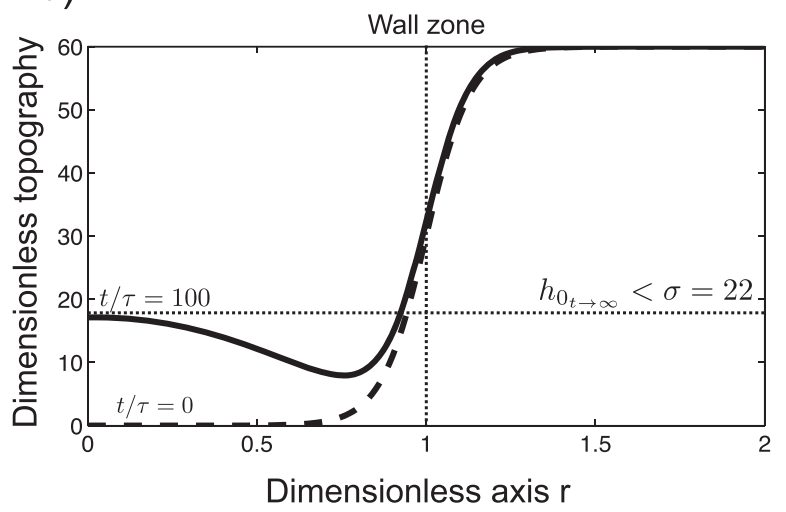

d)

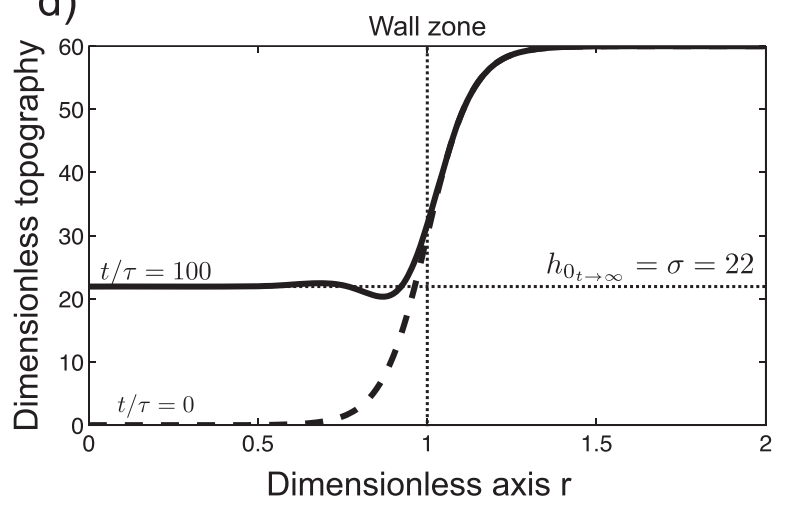

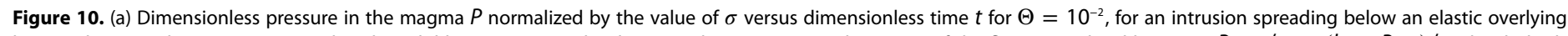

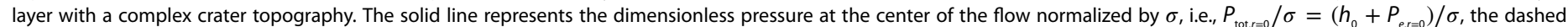

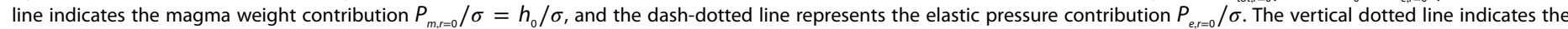

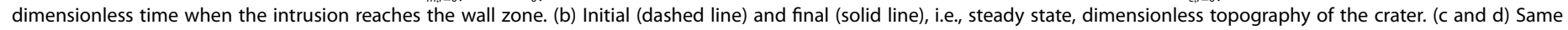

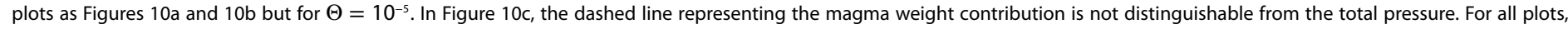
horizontal dotted lines represent asymptotic behaviors. We use $\gamma=0.02, \Xi=50, \zeta=0.13, \Psi=1$, and $\sigma=22$.

For $\Theta=10^{-5}$, the weight of the magma is the dominant contribution to the pressure during the whole flow $\left(P_{m}\right.$ and $P_{\text {tot }}$ are not distinguishable on Figure 10c) and the injection rate remains close to a constant while the intrusion spreads below the crater flat floor in a gravity current regime (Figure 10c). As the intrusion thickens, the weight of magma increases at the center (Figure 10c) leading to a decrease in the total injection rate (32). When, $h_{0}=\sigma=22$, no more magma intrudes below the crater, the intrusion thickness and the radius stabilized, and the intrusion reaches its steady state shape (Figures 10c and 10d).

The normalized pressure head thus controls the amount of shallowing of the crater floor. If the pressure head is larger than the weight of the crust that has been redistributed by cratering, the intrusion would pass the wall zone and reach a constant thickness, but it would still continue to flow laterally beyond the wall zone (see lateral spreading regime in Michaut [2011]). In contrast, if the pressure head is less than the weight of the crust that has been redistributed by cratering, the intrusion reaches a steady state shape and deformations are constrained within the crater. In that case, for small values of $\Theta$, the amount of shallowing is equal to the normalized pressure head $\sigma$, while, for relatively large values of $\Theta$, the intrusion reaches a steady state profile for a thickness $h_{0} \leq \sigma$.

\section{Discussion}

\subsection{Floor Appearance}

Viscous relaxation models are not able to reproduce the amount of shallowing observed at FFCs [Dombard and Gillis, 2001]. Furthermore, viscous relaxation scales with the crater diameter while the shallowing observed at FFCs does not [Schultz, 1976a]. Finally, viscous relaxation cannot explain the presence, next to each other, of large, old, unmodified craters and small, younger FFCs as observed by Schultz [1976a]. 
In contrast, our model is able to reproduce the diversity of floor appearances observed at FFCs and thus confirms the predictions initially made by Schultz [1976a]. In particular, for a given crater size, the thickening caused by the increase in lithostatic pressure at the crater wall zone induces either a convex uplift of the crater floor, if the intrusion occurs below a thick elastic layer, or a piston-like uplift of the crater floor if the intrusion occurs below a strengthless and highly brecciated zone or below a thin elastic layer. This piston-like uplift results in a large, shallowed, plate-like floor as predicted by Schultz [1976a].

In addition, the model shows that the final appearance of the crater floor is controlled by the ratio between the flexural wavelength of the overlying layer that mainly depends on the elastic layer thickness and the crater size, i.e., on the number $\Theta=(\Lambda / C)^{4}$. Indeed, because the deformation of the overlying elastic layer is accommodated along a width of $4 \Lambda$, our model predicts a transition between a flat floor and a convex floor when $4 \Lambda \sim C$, i.e., at $\Theta=10^{-3}$. For large values of $\Theta>10^{-3}$, i.e., small craters and/or a thick elastic layer overlying the intrusion, the bending of the upper elastic layer affects the whole crater width resulting in a convex crater floor (Figure 7a). On the contrary, for large craters and/or a thin elastic layer overlying the intrusion, i.e., small values of $\Theta<10^{-3}$, the thickening of the flat-top intrusion results in a flat central floor with a circular moat that borders the interior of the wall zone (Figure 7b). This circular moat results from the elastic deformation of the overlying layer at the intrusion front, and its size is equal to $4 \Lambda$. Finally, if the overlying layer is strengthless and $\Theta=0$, the crater floor appears flat or slightly concave up because of the density difference between the magma and the overlying layer.

FFCs of classes 3 and 5 show a central uplifted flat floor; they generally have a large diameter: the mean diameter is of $50 \mathrm{~km}$ for craters of class 3, and $70 \mathrm{~km}$ for craters of class 5 (Table 1) [Schultz, 1976a; Jozwiak et al., 2012]. These large diameters are coherent with a small value of $\Theta$ (24), i.e., with an intrusion in the gravity current regime as it reaches the wall zone and a piston-like uplift of the central flat floor of the crater. A moat bordering the wall region is observed for craters of class 3 in agreement with our results for small values of $\Theta$ (see for instance the topography of FFC Warner, Figure 1b). Craters of class 5 have a central shallowed flat floor but do not show a moat bordering the wall region (Table 1). One possibility is that the intrusion has been emplaced below a strengthless layer. Another possibility is that these craters have been partially infilled before deformation by basin ejecta [Schultz, 1976a]; as a result, the thickening of the intrusion was limited and not important enough to form a moat. Furthermore, for large craters and/or intrusions below a thin elastic layer, our model predicts that the maximum stresses are concentrated upon the intrusion edge within an annulus bordering the wall region (See Appendix B, Figure B1c). This stress concentration in front of the crater wall could induce new fractures or reactivate preliminary fractures caused by the impact, providing privileged paths for the magma to rise to the surface [Schultz, 1976a]. Indeed, pyroclastic deposits have been observed in floor-fractured craters of classes 3 and 5, preferentially located within fractures adjacent to the wall zone [Schultz, 1976a].

Convex floors characterize FFCs of classes 2 and 4 . For these craters, the topography and deformations are larger at the center and decrease progressively toward the wall zone, characterizing a maximum in stress and uplift at the center. The resulting central brittle failure at the crater floor sometimes enhances the apparent floor convexity (see, for instance, the well-defined fault that surrounds the central peak of FFC Briggs, Figure 1a). Craters of classes 2 and 4 are in fact generally small; their average diameters are 30 and $20 \mathrm{~km}$ respectively [Jozwiak et al., 2012] with some of them being initially simple craters (Table 1); this is coherent with large values of $\Theta$ and a final convex floor appearance according to our model. In FFCs of class 4, a more or less pronounced narrow $\mathrm{V}$-shaped moat is observed adjacent to the wall zone and pronounced inner ridges for FFCs of class $4 \mathrm{~b}$. In the frame of our model, we can interpret these features as resulting from the rupture of the upper layer during the thickening regime. Indeed, our model predicts that the radial stress might not be maximum at the crater center, even for a convex floor, but at a given radial coordinate $r_{\sigma_{\max }}$ such that $0<r_{\sigma_{\max }}<1$ (see Appendix B, Figures B1a, and B1b) because the intrusion might transition from an elastic to a gravity current shape before the wall zone and because the elastic thickness increases toward the wall zone. The location of $r_{\sigma_{\max }}$ depends on time and on the values of $\Theta$ and $\Psi$. This location of maximum stress provides a preferred location for a concentric failure of the upper elastic layer.

Furthermore, our model can also explain the variable characteristics of central peaks of FFCs [Schultz, 1976a]. In the case where an elastic layer is present, our model predicts that the central peak would be leveled up during the uplift and slightly smaller than initially (see Appendix C, Figures C1b, and C1c). Schultz [1976a] indeed observed that there is a tendency for central peaks of FFCs to be smaller than those for 
well-preserved impact craters. Furthermore, a small circular moat borders the central peak if this elastic layer is relatively thin, such $4 \Lambda \ll C$ (see Appendix $C$ and Figure $C 1 b$ ). The build up of stress resulting from the flexure within this moat could be coherent with the fracture pattern observed in the vicinity of central peaks at some FFCs [Schultz, 1976a]. Finally, if the layer overlying the intrusion is formed by incoherent breccias, the central peak would be buried during the uplift, or its initial size significantly reduced if the shallowing is smaller than the central peak height; it could thus be absent from the uplifted floor, as observed in a few cases [Schultz, 1976a] (see Appendix C and Figure C1a).

\subsection{Depth of the Intrusion}

The deformation of a layer overlying a shallow magmatic intrusion such as a laccolith has been classically modeled by the upward deflection of a thin elastic layer submitted to a pressure $P_{d}$ within the intrusion (29). In this kind of static model, the internal pressure $P_{d}$ within the intrusion is usually assumed to be constant and equal to the hydrostatic pressure due to the weight of the magma at the center of the intrusion. The solution to this problem gives a floor deflection, or maximum intrusion thickness $h_{0}$, that varies with radius to the fourth (29) [Johnson and Pollard, 1973; Pollard and Johnson, 1973]. In the case of an intrusion below the floor of a crater, the crater radius clamps the elastic overlying layer and the intrusion radius can be considered equal to the crater radius, if the deformation affects the whole crater floor. And hence, this approach has motivated many previous studies to derive the intrusion depth from the estimated uplift at a given floor-fractured crater [Wichman and Schultz, 1996; Jozwiak et al., 2012].

However, there is an inconsistency in equating the pressure due to magma weight and the elastic pressure deforming the overlying plate, since both pressures act in favor of flow spreading. Assuming that the final steady state shape of the flow has been reached, i.e., that the intrusion has reached the wall zone and that the pressure above the feeding conduit is equal to the initial overpressure driving the flow in the conduit (see section 4.5), and assuming that the overlying elastic layer making the crater floor has a constant thickness up to the wall zone, the function (29) may indeed describe the vertical displacement of the overlying layer due to an elastic pressure $P_{e}$. But this is true only in the case where the intrusion is in the elastic regime, i.e., if the elastic pressure is dominant over the pressure due to magma weight and in this case, the pressure necessary for deforming the overlying plate $P_{e}$ is then not supported by magma weight but by the driving pressure within the feeding dyke [see Michaut, 2011, section 6.1]. Furthermore, if the thickening at the wall zone is significant, the curvature of the intrusion is accentuated by the interaction with the wall zone and (29) is not accurate anymore. Moreover, for large craters or shallow intrusions, i.e., $4 \Lambda<C$, the intrusion lies in the gravity current regime when it reaches the crater wall zone. In that case, the dominant pressure comes from magma weight and, assuming the intrusion has reached the wall zone and its steady state shape, then the overpressure driving the injection is compensated by magma weight and $P_{d}=\rho_{m} g h$ is a good estimate of the pressure within the flow. However, in this case, the intrusion is characterized by a flat top and its profile is very different from (29) (see section 4.3.1) [Bunger and Cruden, 2011; Michaut, 2011] and hence, (29) is no longer accurate to derive the intrusion depth.

Finally, using (29) and equating $P_{d}=\rho_{m} g h_{0}$ to derive the intrusion depth $T_{e}^{0}$ at FFCs leads to an intrusion depth that scales with the crater diameter to the power four third, i.e., the larger the crater, the deeper the intrusion. The magma intrudes below a brecciated zone whose thickness might indeed increase with impact energy and hence with crater radius; however, the scaling derived above does not deal with the physics of impact and hence cannot provide any information on the brecciated layer thickness. Furthermore, the magma may emplace deeper into the crust.

In contrast, the model we develop provides a simple method to estimate the elastic thickness of the layer overlying the intrusion from the floor appearance. As discussed above, the floor of a crater appears convex if the flexural wavelength of the layer overlying the intrusion is such that $4 \Lambda \geq C$. As $\Lambda$ mainly depends on $T_{e^{\prime}}^{0}$ and assuming the intrusion has reached the crater wall zone, this inequality provides a lower bound for the elastic thickness of the upper layer:

$$
T_{e}^{0} \geq\left(\frac{12\left(1-v^{2}\right) \rho_{m} g C^{4}}{4^{4} E}\right)^{1 / 3}
$$

For instance, Briggs is a class $2 \mathrm{FFC}$ of $37 \mathrm{~km}$ in diameter and shows a topography consistent with a convex uplift of the crater floor (Figure 1a). Hence, (35) applies and we estimate that the intrusion lies beneath an elastic layer whose thickness is equal to or deeper than $1.6 \mathrm{~km}$, using parameter values listed in Table (2). 
This value is only a minimum value for the intrusion depth, as it does not take into account the potential presence of a brecciated layer on top or below the elastic layer.

In the case of a crater showing a flat floor with a circular moat, the intrusion should be in a gravity current regime and assuming it has reached the wall zone (which is likely to be the case if the shallowing is significant), then the width of the moat directly provides an estimate of the flexural wavelength of the overlying elastic layer and hence of the elastic thickness. Indeed, the size of the moat corresponds to the width of the intrusion edge, i.e., to $4 \Lambda$ (see Figures $5 d, 7 b$, and section 4.2). For instance, for the class 3 FFC Warner, which is $35 \mathrm{~km}$ in diameter, the deformation shows a plate-like central flat floor, with a moat bordering the wall region of about one third of the crater radius (Figure $1 \mathrm{~b})$. Hence, $(4 \Lambda) / C \sim 1 / 3$ and the elastic thickness $T_{e}^{0}$ varies between 300 and $600 \mathrm{~m}$. Again, this estimate provides a minimum value for the intrusion depth.

\subsection{Injection Rate and Formation Time Scale}

Earlier works have shown that, for craters smaller than $100 \mathrm{~km}$ in diameter, the shallowing associated with viscous relaxation is small or almost nonexistent [Dombard and Gillis, 2001]. In contrast, our model of magmatic intrusion is able to reproduce the large discrepancy in crater floor shallowing observed at FFCs. Two mechanisms might control the final amount of shallowing: the injection rate and the cooling of the intrusion.

If the cooling time scale of the intrusion is large compared to its formation time scale, then the intrusion reaches the wall zone and thickens. In that case, assuming a constant injection rate, the maximum amount of shallowing corresponds to the intrusion thickness that evolves toward a dimensionless thickness of $\Xi+H_{g}$. However, as explained in section 4.5, the injection rate should decrease as the intrusion grows, in particular during the thickening phase. Indeed, when the magma reaches the wall zone, the pressure due to magma weight and to elastic deformation of the overlying layer increases as the crater floor is uplifted; it might finally compensate for the initial pressure driving the flow in the melt source. An estimate of this initial overpressure can be derived from our model in some cases. Indeed, for small values of $\Theta$, i.e., for craters that show a flat floor like those of classes 3 and 5, the overpressure in the melt source might be estimated from the final thickness of the intrusion at the center $h_{0}^{f}$, i.e., $\Delta P=\rho_{m} g h_{0}^{f}$ as proposed by Jozwiak et al. [2012] (Figures 10c and 10d). However, for large values of $\Theta$, i.e., for craters that show a convex floor, as craters of classes 2 and 4, this assumption is inappropriate as both the pressure due to magma weight and the elastic pressure contribute to compensate for the initial overpressure $\Delta P$, i.e., $\Delta P>\rho_{m} g h_{0}^{f}$ (Figures 10a and 10b).

For most FFCs, the deformation is contained within the crater floor [Schultz, 1976a] suggesting that the removal of material during the impact controlled the initial overpressure in the melt source. However, impact craters commonly show a raised rim, which is uplifted relative to the preimpact surface and this structure could also contribute to prevent the intrusion from growing beyond the wall zone. Finally, in a few cases such as crater Haldane [Schultz, 1976a], the deformations extend well beyond the wall zone and suggest that the intrusion passed the wall zone, i.e., that the initial overpressure might have been larger than $\rho_{c} g D_{c}+P_{r}$, where $P_{r}$ is the lithostatic pressure excess induced by the raised rim.

The deep floor of Class 1 FFCs suggests that these craters did not undergo the thickening phase (Table 1). However, the network of fractures on their floor is coherent with the spreading of an underlying intrusion that might be an aborted sill that cooled before reaching the wall zone. In that case, the time scale for the intrusion to reach the wall zone $\tau=\left(\pi C^{2} H\right) / Q_{0}$ is larger than the time scale for cooling of the intrusion. The time scale for cooling can be estimated from the characteristic time for conductive cooling of an intrusion of average thickness $\bar{h}$

$$
\tau_{c}=\frac{\bar{h}^{2}}{\pi \kappa}
$$

where $\kappa=10^{-6} \mathrm{~m}^{2} \cdot \mathrm{s}^{-1}$ is the thermal diffusivity of basalt. For large craters of class 1 , the intrusion is more likely to be in a gravity current regime with an average thickness close to $\bar{h} \sim 2 H$. The inequality $\tau_{c} \leq \tau$ ((15) and (36)) provides an upper bound for the average injection rate:

$$
Q_{0} \leq 408\left(C^{8} \kappa^{4} \rho_{m} g\right)^{\frac{1}{5}}
$$

For instance, for the crater Atlas, which is $87 \mathrm{~km}$ in diameter and is located at $46.7^{\circ} \mathrm{N}, 44.4^{\circ} \mathrm{E}$ in the southeast of Mare Frigoris, the injection rate should be smaller than $10^{-2} \mathrm{~m}^{3} \mathrm{~s}^{-1}$, using parameter values listed in 
Table (2). This value is, for instance, in agreement with the extrusion rate of lavas on the Moon estimated at the end of the Imbrian and during the Eratosthenian period by Head and Wilson [1992]. However, most of the FFCs have undergone a thickening stage and hence are such that $\tau_{c} \geq \tau$ and this inequality provides a lower bound for the average injection rate below these craters.

\section{Conclusion}

The model shows that the spreading of an intrusion at depth below a crater is able to reproduce the deformations observed at FFCs and thus confirms the different predictions made by Schultz [1976a]. In particular, the increase in lithostatic pressure and flexural wavelength of the overlying layer at the crater wall zone prevents the intrusion from spreading radially and induces a thickening of the intrusion that leads to the shallowing of the crater floor. When the timescale for conductive cooling of the intrusion is large compared to the formation timescale, the amount of shallowing is controlled by the initial overpressure in the melt source. The model predicts that the final appearance of the crater floor, including the presence/disappearance of a central peak, is mainly controlled by the upper layer flexural wavelength $\Lambda$, i.e., its structure and thickness, and the crater radius $C$.

For an overlying layer with no elastic strength, i.e., an intrusion emplaced within or immediately beneath a brecciated lens, the crater floor is flat at the end of the deformation. If an elastic layer lies on top of the intrusion, but is sufficiently thin such that $C \geq 4 \Lambda$, the crater shows a central plate-like floor separated from the wall zone by a circular moat. This moat results from the deformation of the thin elastic overlying layer above the intrusion front, and its width is equal to $4 \Lambda$. The depth of the moat is controlled by the amount of thickening of the overlying layer at the crater wall zone. Finally, if the intrusion is emplaced beneath a thick elastic layer such that $C<4 \Lambda$, the crater shows a convex floor.

Within the framework of this model, FFCs of class 1 would result from aborted sills that have cooled before reaching the wall zone. Craters showing a convex floor, such as those of classes 2 and 4, would result from the spreading of an intrusion below a relatively thick elastic layer, such that $\Lambda \geq C / 4$. On the contrary, craters showing a flat floor, such as those of classes 3 and 5 , would result from the spreading of an intrusion beneath a relatively thin elastic layer, such that $\Lambda<<C / 4$. The wide circular U-shaped moat of class 3 FFCs is well explained by the elastic deformation of the thin elastic layer overlying the intrusion, while the V-shaped moat of class 4 FFCs, showing a convex floor, could be explained by the rupture of the overlying layer, which is predicted to occur at a radial coordinate intermediate between the center and the wall zone. The absence of a moat for craters of class 5 could suggest that the intrusion develops immediately beneath or within a breccia lens which has not been consolidated by impact melt. Finally, class 6 FFCs are filled up by lavas that have reached the surface.

In addition, the model allows constraining the elastic thickness of the layer lying on top of the intrusion, which provides a lower bound for the intrusion depth.

\section{Appendix A: Numerical Scheme}

We use a fully implicit finite-volume method to solve (18). The discretization is obtained by integrating over a finite number of nonoverlapping control volumes, each control volume surrounding one grid point [Patankar, 1980]. The grid is defined by the relation $r_{i}=(i-0.5) \Delta r$ in order to avoid problems at the center. The points $b$ and $a$ define the face of the control volume surrounding $i$ such that $r_{a}=r_{i}+\Delta r / 2$ and $r_{b}=r_{i}-\Delta r / 2$. Because we are using an axisymmetric geometry, the control volume is an annulus of interior radius $r_{b}$ and exterior radius $r_{a}$ and its surface is $S=\pi\left(r_{a}^{2}-r_{b}^{2}\right)$. Integration of (18) over the control volume surrounding $i$ during a time $\Delta t$ gives the following:

$$
\int_{t}^{t+\Delta t} \int_{r_{b}}^{r_{a}} \frac{\partial h^{*}}{\partial t} 2 \pi r \mathrm{~d} r \mathrm{~d} t=\int_{t}^{t+\Delta t} \int_{b}^{a} \Phi(r, t) 2 \pi r \mathrm{~d} r \mathrm{~d} t
$$

where $\Phi(r, t)$ stands for the right-hand side of (18). The classical second-order $\left(\propto \Delta r^{2}\right)$ approximations is taken to derive the successive space derivatives (i.e., $\left.\left.\frac{\partial \Phi(r)}{\partial r}\right|_{r_{a}}=\frac{\Phi(i+1)-\Phi(i)}{\Delta r}\right)$. In this way, we ensure that our final scheme is of second order. However, for more precision, the elastic pressure is calculated using a fourth-order scheme (see section A1.3). In the following, we derive each term of the right-hand side of (18) separately, $h$ refers to the value of the thickness at a time $t+\Delta t$ and $h^{n}$ to the value at a time $t$. 


\section{A1. Discretization}

1. Time Derivative To discretize the time derivative, we shall consider that the value of the grid point $h_{i}$ prevails throughout the control volume such that

$$
\int_{t}^{t+\Delta t} \int_{r_{b}}^{r_{a}} \frac{\partial h^{*}}{\partial t} 2 \pi r d r d t=\left(h_{i}-h_{i}^{n}\right) S
$$

\section{Gravitational Term}

$$
\begin{array}{r}
\int_{t}^{t+\Delta t} \int_{r_{b}}^{r_{a}} \frac{1}{r^{*}} \frac{\partial}{\partial r^{*}}\left(r^{*} h^{3} \frac{\partial h}{\partial r^{*}}\right) 2 \pi r \mathrm{~d} r \mathrm{~d} t \\
=A_{i}^{g} h_{i+1}+B_{i}^{g} h_{i-1}-\left(A_{i}^{g}+B_{i}^{g}\right) h_{i}
\end{array}
$$

with $A_{i}^{g}=A=\left(2 \pi \Delta t r_{a} h_{a}^{3}\right) / \Delta r$ and $B_{i}^{g}=B=\left(2 \pi \Delta t r_{b} h_{b}^{3}\right) / \Delta r$, where the value of $h_{a}^{3}$ (respectively, $\left.h_{b}^{3}\right)$ is approximated by $\left(h_{i+1}^{3}+h_{i}^{3}\right) / 2$ (respectively, $\left.\left(h_{i}^{3}+h_{i-1}^{3}\right) / 2\right)$.

\section{Elastic Term}

$$
\begin{array}{r}
\int_{t}^{t+\Delta t} \int_{r_{b}}^{r_{a}} \Theta \frac{1}{r^{*}} \frac{\partial}{\partial r^{*}}\left(r^{*} h^{3} \frac{\partial P_{e}}{\partial r^{*}}\right) 2 \pi r d r d t \\
=A_{i}^{e} P_{e, i+1}+B_{i}^{e} P_{e, i-1}-\left(A_{i}^{e}+B_{i}^{e}\right) P_{e, i}
\end{array}
$$

where $A_{i}^{e}=\Theta A, B_{i}^{e}=\Theta B$, and $P_{e}=\nabla_{r}^{2}\left(\Pi(r) \nabla_{r}^{2} h(r)\right)$, with $\Pi(r)=(1+\Psi \xi(r))^{3}$ is the dimensionless elastic pressure which is discretized using a fourth-order finite difference scheme:

$$
P_{e, i}=\alpha_{i} h_{i-3}+\beta_{i} h_{i-2}+\gamma_{i} h_{i-1}+\lambda_{i} h_{i}+\kappa_{i} h_{i+1}+\delta_{i} h_{i+2}+\epsilon_{i} h_{i+3}
$$

with

$$
\begin{aligned}
\alpha_{i} & =\frac{1}{24 \Delta r^{4}}\left(-4 p_{4}+3 p_{3} \Delta_{r}\right) \\
\beta_{i} & =\frac{1}{24 \Delta r^{4}}\left(48 p_{4}-24 p_{3} \Delta_{r}-2 p_{2} \Delta_{r}^{2}+2 p_{1} \Delta_{r}^{3}\right) \\
\gamma_{i} & =\frac{1}{24 \Delta r^{4}}\left(-156 p_{4}+39 p_{3} \Delta_{r}+32 p_{2} \Delta_{r}^{2}-16 p_{1} \Delta_{r}^{3}\right) \\
\lambda_{i} & =\frac{1}{24 \Delta r^{4}}\left(224 p_{4}-60 p_{2} \Delta r^{2}\right) \\
\kappa_{i} & =\frac{1}{24 \Delta r^{4}}\left(-156 p_{4}-39 p_{3} \Delta_{r}+32 p_{2} \Delta_{r}^{2}+16 p_{1} \Delta_{r}^{3}\right) \\
\delta_{i} & =\frac{1}{24 \Delta r^{4}}\left(48 p_{4}+24 p_{3} \Delta_{r}-2 p_{2} \Delta_{r}^{2}-2 p_{1} \Delta_{r}^{3}\right) \\
\epsilon_{i} & =\frac{1}{24 \Delta r^{4}}\left(-4 p_{4}-3 p_{3} \Delta_{r}\right)
\end{aligned}
$$

where

$$
\begin{aligned}
& p_{1}=\frac{\Pi_{i}^{\prime \prime}}{r_{i}}-\frac{\Pi_{i}^{\prime}}{r_{i}^{2}}+\frac{\Pi}{r_{i}^{3}} \\
& p_{2}=\Pi_{i}^{\prime \prime}+\frac{3 \Pi_{i}^{\prime}}{r_{i}}+\frac{\Pi_{i}}{r_{i}^{2}} \\
& p_{3}=2 \Pi_{i}^{\prime}+\frac{2 \Pi_{i}}{r_{i}} \\
& p_{4}=\Pi_{i}
\end{aligned}
$$

and where $\Pi_{i}=\left(1+\Psi \xi_{i}\right)^{3}$ and $\Pi_{i}^{\prime}$ and $\Pi_{i}^{\prime \prime}$ are its first and second derivatives with respect to the radial coordinate.

\section{Hydrostatic Term}

$$
\begin{array}{r}
S_{i}^{h}=\int_{t}^{t+\Delta t} \int_{r_{b}}^{r_{a}} \Xi \frac{1}{r^{*}} \frac{\partial}{\partial r^{*}}\left(r^{*} h^{3} \frac{\partial \xi}{\partial r}\right) 2 \pi r d r \\
=U^{h}\left(\left.r_{a} h_{a}^{3} \frac{\partial \xi}{\partial r}\right|_{a}-\left.r_{b} h_{b}^{3} \frac{\partial \xi}{\partial r}\right|_{b}\right)
\end{array}
$$

where $U^{h}=2 \pi \Xi \Delta t$. 


\section{Injection Term}

$$
\begin{aligned}
S_{i}^{i} & =\int_{t}^{t+\Delta t} \int_{r_{b}}^{r_{a}} \frac{32}{\gamma^{2}}\left(\frac{1}{4}-\frac{r^{2}}{\gamma^{2}}\right) 2 \pi r d r d t \\
& =U^{i}\left(\gamma^{2}-2\left(r_{a}^{2}+r_{b}^{2}\right)\right)
\end{aligned}
$$

where $U^{i}=\frac{85 \Delta t}{\gamma^{4}}$.

\section{Implicit Scheme}

Substituting (A2), (A3), (A4), (A6), and (A7) in (A1) and injecting (A5), we get the final scheme given by the following equation:

$$
a_{i} h_{i-4}+b_{i} h_{i-3}+c_{i} h_{i-2}+d_{i} h_{i-1}+e_{i} h_{i}+f_{i} h_{i+1}+g_{i} h_{i+2}+k_{i} h_{i+3}+l_{i} h_{i+4}=J_{i}
$$

where the different coefficients are defined by

$$
\begin{gathered}
a_{i}=-B_{i}^{e} \alpha_{i-1} \\
b_{i}=\left(B_{i}^{e}+A_{i}^{e}\right) \alpha_{i}-B_{i}^{e} \beta_{i-1} \\
c_{i}=\left(B_{i}^{e}+A_{i}^{e}\right) \beta_{i}-B_{i}^{e} \gamma_{i-1}-A_{i}^{e} \alpha_{i+1} \\
d_{i}=\left(B_{i}^{e}+A_{i}^{e}\right) \gamma_{i}-B_{i}^{e} \lambda_{i-1}-A_{i}^{e} \beta_{i+1}-B^{g} \\
e_{i}=S+\left(B_{i}^{e}+A_{i}^{e}\right) \lambda_{i}-B_{i}^{e} \kappa_{i-1}-A_{i}^{e} \gamma_{i+1}+B^{g}+A^{g} \\
f_{i}=\left(B_{i}^{e}+A_{i}^{e}\right) \kappa_{i}-B_{i}^{e} \delta_{i-1}-A_{i}^{e} \lambda_{i+1}-A^{g} \\
g_{i}=\left(B_{i}^{e}+A_{i}^{e}\right) \delta_{i}-B_{i}^{e} \epsilon_{i-1}-A_{i}^{e} \kappa_{i+1} \\
k_{i}=\left(B_{i}^{e}+A_{i}^{e}\right) \epsilon_{i}-A_{i}^{e} \delta_{i+1} \\
I_{i}=-A_{i}^{e} \epsilon_{i+1} \\
J_{i}=\left(S h_{i}^{n}+S_{i}^{i}+S_{i}^{h}\right)
\end{gathered}
$$

\section{A2. Boundary Conditions}

Since the flow is symmetric in $r=0$, we require that

$$
\begin{aligned}
& \left.\frac{\partial h}{\partial r}\right|_{r=0}=0 \\
& \left.\frac{\partial P_{e}}{\partial r}\right|_{r=0}=0
\end{aligned}
$$

Boundary conditions at the front of the intrusion are accounted for by using a grid much larger than the intrusion where $h=0$ beyond the flow.

\section{A3. Algorithm}

The fully implicit discretization (A8) can be rewritten as a linear system $\Omega\left(h^{3}\right) \bar{h}=\bar{J}$ where $\bar{h}$ is a vector containing the value of $h$ a $t+\Delta t$ and $\bar{J}$ containing the right-hand side of (A8). The matrix $\Omega\left(h^{3}\right)$ is a septadiagonal matrix and is solved by using a septadiagonal algorithm. However, due to the nonlinearity of the problem (i.e., the coefficients $A_{e}, B_{e}, A_{g}, B_{g}$, and $S^{h}$ within the matrix $\Omega\left(h_{i}^{3}\right)$ and $\bar{J}$ depend on $h_{i}^{3}$ ), we first have to assume values for $h_{i}$ at each grid point to invert for the matrix and get the value of $h$ at $t+\Delta t$. We use the following iterative scheme:

1. Start with a guess at all grid point for $h_{i}=h_{i}^{n}$.

2. Calculate tentative values for the different coefficients of the system (nonlinear terms).

3. Apply the septadiagonal matrix algorithm to solve (A8) and get a new value of $h_{i}$.

4. With this new $h_{i}$, we return to step 2 and repeat steps 2 to 4 until further repetitions cease to produce any significant change in $h_{i}$ (i.e., $\left|h_{i}^{\text {new }}-h_{i}\right|<\epsilon$ where $\epsilon=10^{-4}$ ).

The final unchanging state is considered as the solution for the thickness of the flow at $t+\Delta t$. 
a) $\Theta=10^{-2}$
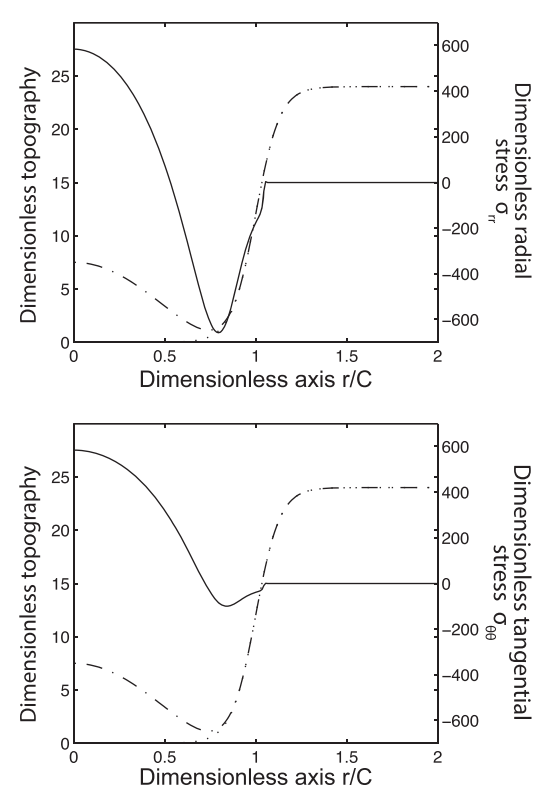

b) $\Theta=10^{-3}$
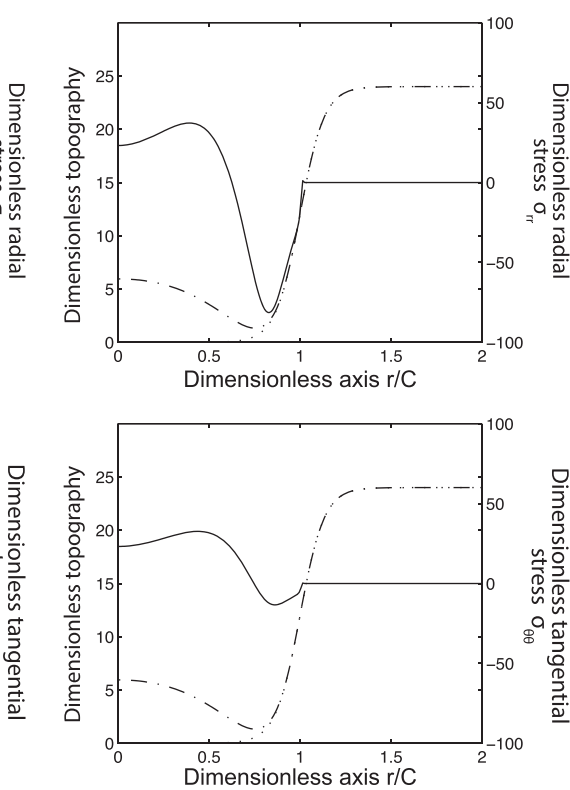

c) $\Theta=10^{-5}$
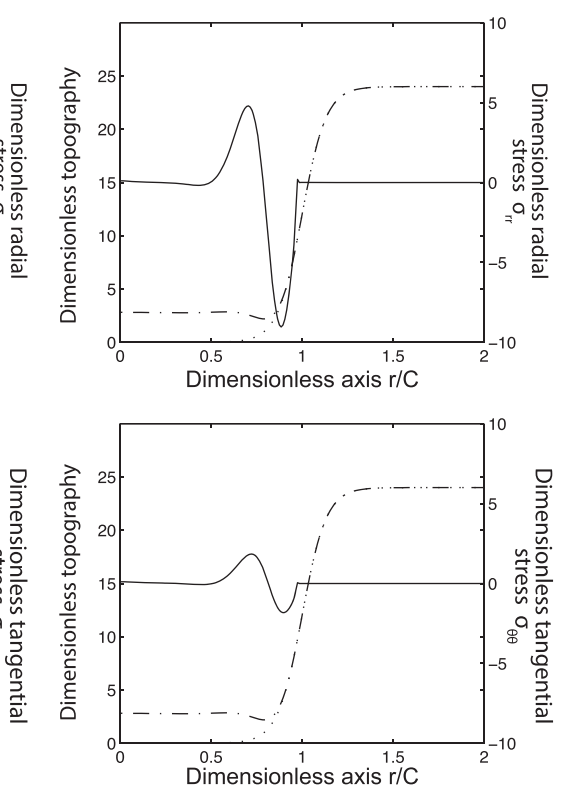

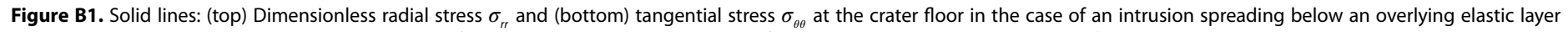

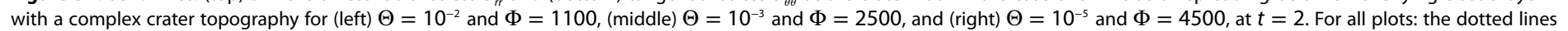

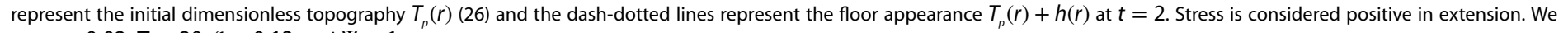
use $\gamma=0.02, \Xi=20, \zeta=0.13$, and $\Psi=1$.

\section{Appendix B: Elastic Stresses in the Upper Elastic Layer}

The stress conditions within the crater floor can be approximated using the small displacement theory. In an axisymmetric geometry, the small strain-displacement equations at the surface are

$$
\begin{gathered}
\epsilon_{r r}=\frac{\partial u_{r}}{\partial r}=-\frac{T_{e}(r)}{2} \frac{\partial^{2} h}{\partial r^{2}} \\
\epsilon_{\theta \theta}=\frac{u_{r}}{r}=-\frac{T_{e}(r)}{2 r} \frac{\partial h}{\partial r}
\end{gathered}
$$

Hence, the stress conditions at the surface are given by Hooke's laws for a material under plane stress:

$$
\begin{gathered}
\sigma_{r r}=-\frac{E T_{e}(r)}{2\left(1-v^{2}\right)}\left(\frac{\partial^{2} h}{\partial r^{2}}+\frac{v}{r} \frac{\partial h}{\partial r}\right) \\
\sigma_{\theta \theta}=-\frac{E T_{e}(r)}{2\left(1-v^{2}\right)}\left(\frac{1}{r} \frac{\partial h}{\partial r}+v \frac{\partial^{2} h}{\partial r^{2}}\right)
\end{gathered}
$$

These equations are made dimensionless using the scaling of section 3.3 where the pressure scale is $\rho_{m} g H$. Dimensionless radial and tangential stresses become

$$
\begin{gathered}
\sigma_{r r}=-\Theta \Phi(1+\Psi \xi(r))\left(\frac{\partial^{2} h}{\partial r^{2}}+\frac{v}{r} \frac{\partial h}{\partial r}\right) \\
\sigma_{\theta \theta}=-\Theta \Phi(1+\Psi \xi(r))\left(\frac{1}{r} \frac{\partial h}{\partial r}+v \frac{\partial^{2} h}{\partial r^{2}}\right)
\end{gathered}
$$

where $\xi(r)$ is given by (19) and $\Phi$ is a dimensionless number given by

$$
\Phi=\frac{6}{\left(1-v^{2}\right)}\left(\frac{C}{T_{e}^{0}}\right)^{2}
$$




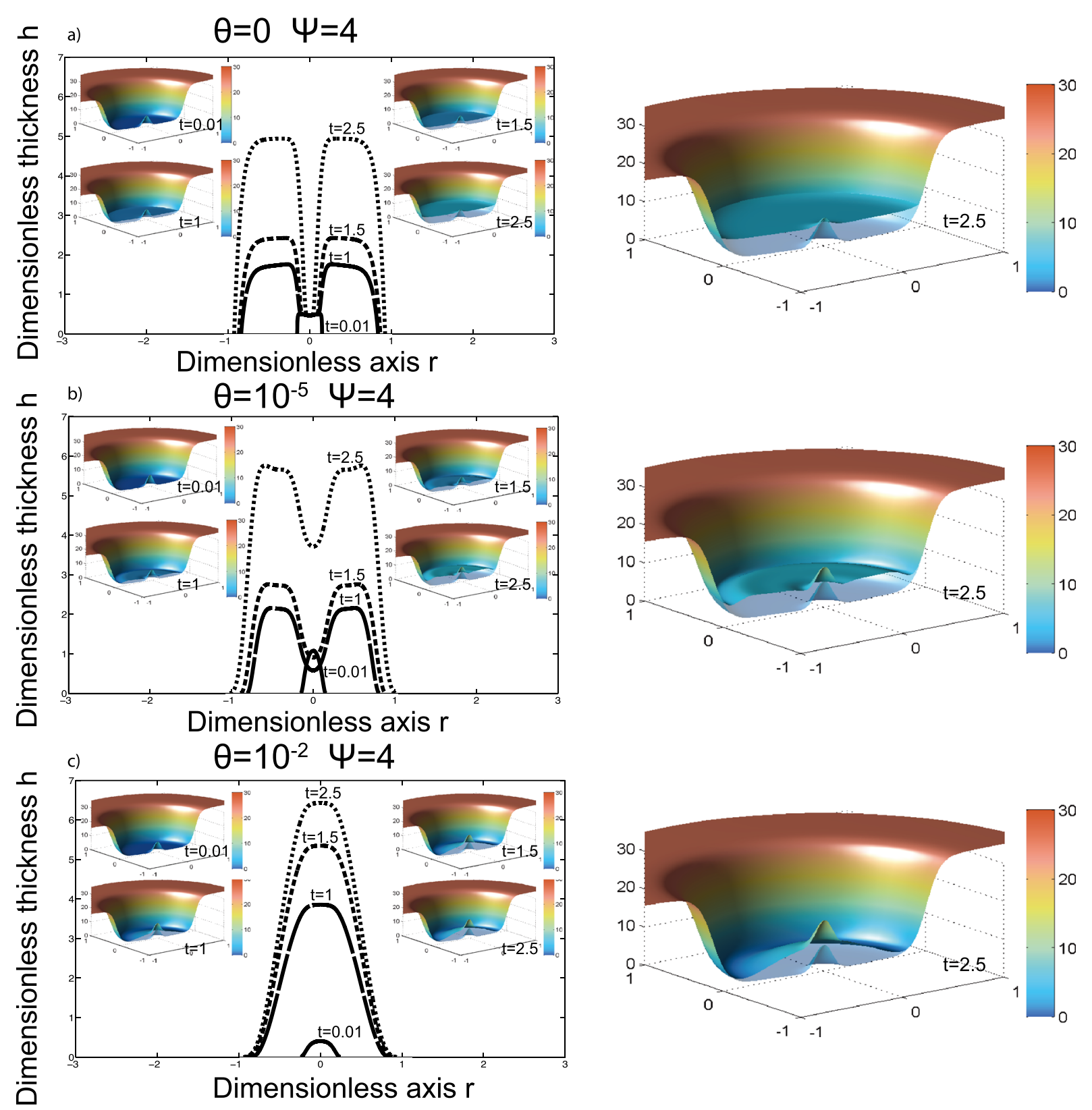

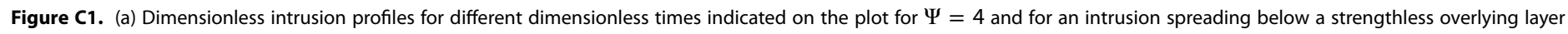

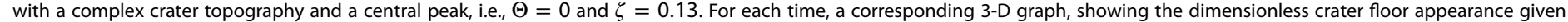

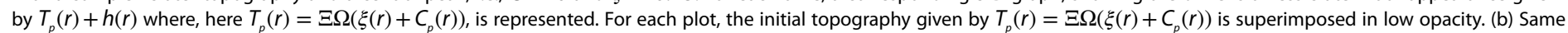
plot but for an overlying elastic layer such that $\Theta=10^{-5}$. (c) Same plot but for an elastic overlying layer such that $\Theta=10^{-2}$. Here we use $\gamma=0.02, \Xi=20$, and $\Psi=4$.

The locations of maximum stress, where fractures are the most likely to initiate, depend on the number $\Theta$ (Figures B1a, B1b, and B1C). If the number $\Theta$ is such that $4 \Lambda>>C$, i.e., $\Theta>10^{-3}$, the intrusion reaches the wall zone in an elastic regime and the maximum stress is at the center (Figure B1a). For $\Theta \sim 10^{-3}, 4 \Lambda \sim C$ and the transition to a gravity current regime occur at the crater wall zone. In that case, the floor is still convex, but the area of maximum stress is located within an annulus at a given coordinate $r_{\sigma_{\max }}$ intermediate between the center and the wall zone, i.e., $0<r_{\sigma_{\max }}<1$ (Figure B1b). Radial and tangential stresses are of the same order of magnitude. For a large crater or a shallow intrusion, i.e., a small value of the number $\Theta<10^{-3}$, the maximum stresses are concentrated within an annulus adjacent to the wall zone upon the intrusion edge where the elastic deformation is important (Figure B1c). The radial stresses that develop at the surface are generally larger than the tangential stresses favoring a circular mode of fracturing. 


\section{Appendix C: Central Peak}

Central peaks induce an increase in the lithostatic pressure and an increase in the overlying layer elastic thickness directly above the intrusion center. Herein we consider an extreme case where the central peak height is one third of the crater depth and its width is one fourth of the crater size by introducing an extra Gaussian function into the elastic thickness expression:

$$
T_{e}(r)=T_{e}^{0}\left(1+\Psi\left(\xi(r)+C_{p}(r)\right)\right)
$$

with

$$
C_{p}(r)=50\left(\frac{0.07}{4}\right)^{2} \exp \left(-\frac{r^{2}}{2\left(\frac{0.07}{4}\right)^{2}}\right)
$$

For a strengthless overlying layer and $\Theta=0$ (section 4.1 equation (27)), the central peak only adds an excess in lithostatic pressure at the center of the crater floor. In response, the intrusion preferentially develops around the central peak and then spreads until it reaches the crater wall (Figure C1a). At the crater wall, the lithostatic pressure increase induces the thickening of the intrusion. However, due to the excess of lithostatic pressure at the center, the center of the intrusion below the central peak does not thicken and the thickening only concerns an annulus located in between the central peak and the crater wall (Figure C1a). At the surface, the central peak height decreases until the thickening is important enough to compensate for the initial excess in lithostatic pressure. A balance between the two pressures gives the final height of the central peak, equal to the initial height times $\left(\rho_{m}-\rho_{c}\right) / \rho_{m}$ (Figure C1a). Next, the resulting central peak is just leveled up with the whole crater floor.

For an elastic overlying layer such that $\Theta=10^{-5}$, the inner part of the intrusion adjacent to the central peak is bent by the weight of the central peak. As a consequence, during the thickening stage, a second circular moat, whose size is $4 \Lambda$, arises and borders the central peak. As previously mentioned, the central peak height decreases until the sum of the elastic and hydrostatic pressure compensate for the initial excess of lithostatic pressure due to the central peak and is then leveled up during floor uplift.

Finally, in the case of a thick elastic overlying layer, i.e., a large value of $\Theta$, the flexural wavelength is almost not affected by the presence of the central peak and the central peak is leveled up with the convex floor during crater floor uplift (Figure C1c).

Acknowledgments

The authors are grateful to R. W. Wichman and P. H. Schultz for their thoughtful comments and careful reviews. This work was supported by PNP/INSU and by Labex UNIVEARTH, Université Paris Diderot.

\section{References}

Baker, D. M. H., J. W. Head, G. A. Neumann, D. E. Smith, and M. T. Zuber (2012), The transition from complex craters to multi-ring basins on the Moon: Quantitative geometric properties from Lunar Reconnaissance Orbiter Lunar Orbiter Laser Altimeter (LOLA) data, J. Geophys. Res., 117, E00H16, doi:10.1029/2011JE004021.

Bray, V. J., G. S. Collins, J. V. Morgan, and P. M. Sschenk (2008), The effect of target properties on crater morphology: Comparison of central peak craters on the Moon and Ganymede, Meteorit. Planet. Sci., 43(12), 1979-1992, doi:10.1111/j.1945-5100.2008.tb00656.x.

Bunger, A. P., and A. R. Cruden (2011), Modeling the growth of laccoliths and large mafic sills: Role of magma body forces, J. Geophys. Res., 116, B02203, doi:10.1029/2010JB007648.

Crisp, J. A. (1984), Rates of magma emplacement and volcanic output, J. Volcanol. Geotherm. Res., 20(3), 177-211, doi:10.1016/0377-0273(84)90039-8.

Dombard, A. J., and J. J. Gillis (2001), Testing the viability of topographic relaxation as a mechanism for the formation of lunar floor-fractured craters, J. Geophys. Res., 106(E11), 27,901-27,909, doi:10.1029/2000JE001388.

El-Baz, F. (1970), Lunar igneous intrusions, Science, 167(3914), 49-50.

Glotch, T. D., et al. (2010), Highly silicic compositions on the Moon, Science, 329(5998), 1510-1513, doi:10.1126/science.1192148.

Hall, J. L., S. C. Solomon, and J. W. Head (1981), Lunar floor-fractured craters: Evidence for viscous relaxation of crater topography, J. Geophys. Res., 86(B10), 9537-9552, doi:10.1029/JB086iB10p09537.

Head, J. W., and L. Wilson (1992), Lunar mare volcanism: Stratigraphy, eruption conditions, and the evolution of secondary crusts, Geochim. Cosmochim. Acta, 56(6), 2155-2175, doi:10.1016/0016-7037(92)90183-J.

Head, J. W., et al. (2009), Volcanism on Mercury: Evidence from the first MESSENGER flyby for extrusive and explosive activity and the volcanic origin of plains, Earth Planet. Sci. Lett., 285(3), 227-242, doi:10.1016/j.epsl.2009.03.007.

Hiesinger, H., and J. W. Head (2006), New views of lunar geoscience: An introduction and overview, Rev. Mineral. Geochem., 60(1), 1-81, doi:10.2138/rmg.2006.60.1.

Huppert, H. E. (1982), The propagation of two-dimensional and axisymmetric viscous gravity currents over a rigid horizontal surface, J. Fluid Mech., 121(1), 43-58, doi:10.1017/S0022112082001797.

Johnson, A. M., and D. D. Pollard (1973), Mechanics of growth of some laccolithic intrusions in the Henry mountains, Utah, I, Tectonophysics, 18(3), 261-309, doi:10.1016/0040-1951(73)90050-4.

Jolliff, B. L., L. R. Gaddis, G. Ryder, C. R. Neal, C. K. Shearer, R. C. Elphic, J. R. Johnson, L. P. Keller, R. L. Kerotev, and D. J. Lawrence (2000), New views of the Moon: Improved understanding through data integration, Eos Trans. AGU, 81(31), 349-355. 
Jozwiak, L. M., J. W. Head, M. T. Zuber, D. E. Smith, and G. A. Neumann (2012), Lunar floor-fractured craters: Classification, distribution, origin and implications for magmatism and shallow crustal structure, J. Geophys. Res., 117, E11005, doi:10.1029/2012JE004134.

Kalynn, J., C. L. Johnson, G. R. Osinski, and O. Barnouin (2013), Topographic characterization of lunar complex craters, Geophys. Res. Lett., 40, 38-42, doi:10.1029/2012GL053608.

Laneuville, M., M. A. Wieczorek, D. Breuer, and N. Tosi (2013), Asymmetric thermal evolution of the Moon, J. Geophys. Res. Planets, 118, 1435-1452, doi:10.1002/jgre.20103.

Melosh, H. J. (1989), Impact Cratering: A Geologic Process, Research supported by NASA, Oxford Monographs on Geology and Geophysics, Oxford University Press, New York. No. 11, 253 p., 1.

Michaut, C. (2011), Dynamics of magmatic intrusions in the upper crust: Theory and applications to laccoliths on Earth and the Moon, J. Geophys. Res., 116, B05205, doi:10.1029/2010JB008108.

Michaut, C., and D. Bercovici (2009), A model for the spreading and compaction of two-phase viscous gravity currents, J. Fluid Mech., 630, 299-329, doi:10.1017/S0022112009006612.

Michaut, C., D. Baratoux, and C. Thorey (2013), Magmatic intrusions and deglaciation at mid-latitude in the northern plains of Mars, Icarus, 225(1), 602-613, doi:10.1016/j.icarus.2013.04.015.

O'Keefe, J. D., and T. J. Ahrens (1999), Complex craters: Relationship of stratigraphy and rings to impact conditions, J. Geophys. Res., 104 (E11), 27,091-27,104, doi:10.1029/1998JE000596.

Patankar, S. V. (1980), Numerical Heat Transfer and Fluid Flow, Series in computational and physical processes in mechanics and thermal sciences, Hemisphere Publishing Company, New York.

Pike, R. J. (1974), Depth/diameter relations of fresh lunar craters: Revision from spacecraft data, Geophys. Res. Lett., 1(7), 291-294, doi:10.1029/GL001i007p00291.

Pike, R. (1976), Crater dimensions from Apollo data and supplemental sources, Moon, 15(3-4), 463-477.

Pike, R. J. (1980), Formation of complex impact craters: Evidence from Mars and other planets, Icarus, 43(1), 1-19, doi:10.1016/0019-1035(80)90083-4.

Pollard, D. D., and A. M. Johnson (1973), Mechanics of growth of some laccolithic intrusions in the Henry mountains, Utah, II: Bending and failure of overburden layers and sill formation, Tectonophysics, 18(3), 311-354, doi:10.1016/0040-1951(73)90051-6.

Rivalta, E., M. Böttinger, and T. Dahm (2005), Buoyancy-driven fracture ascent: Experiments in layered gelatine, J. Volcanol. Geotherm. Res., 144(1-4), 273-285, doi:10.1016/j.jvolgeores.2004.11.030.

Sato, H., K. Kurita, and D. Baratoux (2010), The formation of floor-fractured craters in Xanthe Terra, Icarus, 207(1), 248-264, doi:10.1016/j.icarus.2009.10.023.

Schultz, P. H. (1976a), Floor-fractured lunar craters, Moon, 15(3-4), 241-273, doi:10.1007/BF00562240.

Schultz, P. H. (1976b), Moon Morphology: Interpretations Based on Lunar Orbiter Photography, vol. 1, University of Texas Press, Austin.

Schultz, P. H. (1978), Martian intrusions: Possible sites and implications, Geophys. Res. Lett., 5(6), 457-460, doi:10.1029/GL005i006p00457.

Schultz, P. H. (1988), Cratering on Mercury: A relook, in Mercury, pp. 274-335, Univ. of Ariz. Press, Tucson, Ariz.

Schultz, P. H., and H. Glicken (1979), Impact crater and basin control of igneous processes on Mars, J. Geophys. Res., 84(B14), 8033-8047, doi:10.1029/JB084iB14p08033.

Schultz, P. H., C. Koeberl, T. Bunch, J. Grant, and W. Collins (1994), Ground truth for oblique impact processes: New insight from the Rio Cuarto, Argentina, crater field, Geology, 22(10), 889-892.

Taisne, B., and S. Tait (2009), Eruption versus intrusion? Arrest of propagation of constant volume, buoyant, liquid-filled cracks in an elastic, brittle host, J. Geophys. Res., 114, B06202, doi:10.1029/2009JB006297.

Turcotte, D. L., R. J. Willemann, W. F. Haxby, and J. Norberry (1981), Role of membrane stresses in the support of planetary topography, J. Geophys. Res., 86(B5), 3951-3959, doi:10.1029/JB086iB05p03951.

Walker, G. (1989), Gravitational (density) controls on volcanism, magma chambers and intrusions, Aust. J. Earth Sci., 36(2), 149-165, doi:10.1080/08120098908729479.

Wichman, R. W., and P. H. Schultz (1993), Floor-fractured crater models of the Sudbury Structure, Canada: Implications for initial crater size and crater modification, Meteoritics, 28(2), 222-231, doi:10.1111/j.1945-5100.1993.tb00760.x.

Wichman, R. W., and P. H. Schultz (1995a), Floor-fractured impact craters on Venus: Implications for igneous crater modification and local magmatism, J. Geophys. Res., 100(E2), 3233-3244, doi:10.1029/94JE03206.

Wichman, R. W., and P. H. Schultz (1995b), Floor-fractured craters in Mare Smythii and west of Oceanus Procellarum: Implications of crater modification by viscous relaxation and igneous intrusion models, J. Geophys. Res., 100(E10), 21,201-21,218, doi:10.1029/95JE02297.

Wichman, R. W., and P. H. Schultz (1996), Crater-centered laccoliths on the Moon: Modeling intrusion depth and magmatic pressure at the Crater Taruntius, Icarus, 122(1), 193-199, doi:10.1006/icar.1996.0118.

Wieczorek, M. A., M. T. Zuber, and R. J. Phillips (2001), The role of magma buoyancy on the eruption of lunar basalts, Earth Planet. Sci. Lett., 185(1), 71-83, doi:10.1016/S0012-821X(00)00355-1.

Wieczorek, M. A., et al. (2013), The crust of the Moon as seen by GRAIL, Science, 339(6120), 671-675, doi:10.1126/science.1231530. Wilhelms, D. E., J. F. McCauley, and N. J. Trask (1987), The geologic history of the Moon. US Geological Survey Professional Paper 1348. Wilson, L., and J. W. Head (1981), Ascent and eruption of basaltic magma on the Earth and Moon, J. Geophys. Res., 86(B4), 2971-3001, doi:10.1029/JB086iB04p02971. 